\title{
Magnetic bead handling on-chip: new opportunities for analytical applications
}

\begin{abstract}
This review describes recent advances in the handling and manipulation of magnetic particles in microfluidic systems. Starting from the properties of magnetic nanoparticles and microparticles, their use in magnetic separation, immuno-assays, magnetic resonance imaging, drug delivery, and hyperthermia is discussed. We then focus on new developments in magnetic manipulation, separation, transport, and detection of magnetic microparticles and nanoparticles in microfluidic systems, pointing out the advantages and prospects of these concepts for future analysis applications.
\end{abstract}

Keywords Microfluidics - Magnetic bead handling Magnetic bead assay $\cdot$ Magnetic bead labeling $\cdot$ Magnetic supraparticle structure

\section{Introduction}

Since the introduction of the concept of micro total analysis systems, or $\mu$ TAS, in 1990 (Manz et al. 1990), multiple technologies for the realization of fluidic microsystems have been developed (see, for example, the book of Madou (2002) and the review of Reyes et al. (2002)). Reviews of various fluidic operations in microfluidic systems, such as sample preparation, sample injection, sample manipulation, reaction, separation, and detection, published in the period between 1998 and 2004 were presented by Auroux et al. (2002) and Vilkner et al. (2004). Three of the most important advantages of using microfluidic systems of reduced dimension for analytical applications are known to be: (1) the possibility of using minute quantities of sample and reagents (down to picoliters); (2) relatively fast reaction times when molecular

M. A. M. Gijs

Institute of Microelectronics and Microsystems,

Swiss Federal Institute of Technology Lausanne,

1015 Lausanne EPFL, Switzerland

E-mail: martin.gijs@epfl.ch

Tel.: + 41-21-6936734

Fax: + 41-21-6935950 diffusion lengths are of the order of the microchannel dimension; and (3) a large surface-to-volume ratio, offering an intrinsic compatibility between the use of a microfluidic system and surface-based assays.

Parallel to the boom of microfluidic systems, nanomaterials and nanoparticles have become a hot topic in research. Functional nanoparticles and microparticles ("beads") prepared, for example, by emulsion polymerization or dispersion polymerization, offer a large specific surface for chemical binding, and a polymer colloid or microsphere solution has a low viscosity compared to solutions having the same amount of solid, giving it special properties. Such small particles can be advantageously used as a "mobile substrate" for bio-assays, or even for in vivo applications; they can be easily recovered from a dispersion, reversibly re-dispersed, etc. Several reviews on the preparation and use of polymer particles and polymer colloids for medical, biological, and optical applications exist (Kruis et al. 1998; Kawaguchi 2000). Verpoorte (2003) recently reviewed newly developed technologies for the realization of nanoparticle and microparticle suspension handling systems in general, with a focus on the integration of these applications within microfluidic systems. With respect to open microchannels, microfluidic structures with packed beds of functionalized beads or containing bead suspensions profit from an even larger surface-to-volume ratio, an enhanced interaction of reactive surfaces with passing fluids, and an improved recuperation of reaction products.

Magnetic nanoparticles and microparticles offer still an additional advantage: having embedded magnetic entities, they can be magnetically manipulated using permanent magnets or electromagnets, independently of normal microfluidic or biological processes ${ }^{1}$. This extra degree of freedom is the basis of a still improved

\footnotetext{
${ }^{1}$ It is known that some micro-organisms and animals use geomagnetic field information for orientation. They contain biomineralized magnetite $\left[\mathrm{Fe}_{3} \mathrm{O}_{4}\right]$ particles that can interact with the geomagnetic field, monitor its direction, and are part of a highly sophisticated sensory system.
} 
exposure of the functionalized bead surface to the surrounding liquid and of higher sample pre-concentration efficiencies, due to the increased relative motion of the bead with respect to the fluid.

\section{Physics of magnetic bead manipulation}

Pankhurst et al. (2003) reviewed the applications of magnetic nanoparticles in biomedicine, with a focus on the underlying physics. Of particular interest are small mono-domain nanoparticles. They are single domain because they have a dimension that is typically of the order or smaller than the typical thickness of a magnetic domain wall $\delta=\sqrt{\frac{J S^{2} \pi^{2}}{K a}}$, with $J$ being the magnetic exchange constant, $S$ the total spin quantum number of each atom, $a$ the inter-atomic spacing, and $K$ the magnetic anisotropy constant of the magnetic material (Chikazumi 1964). For iron, assuming that $S=1$, and with $J=2.16 \times 10^{-21} \mathrm{~J}, a=2.86 \times 10^{-10} \mathrm{~m}$ and $K=4.2 \times 10^{4} \mathrm{~J} / \mathrm{m}^{3}$, one calculates a domain wall width of $42 \mathrm{~nm}$. Leslie-Pelecky and Rieke (1996) have reviewed the relation between the morphology of nanostructured materials and their magnetic properties. The time over which the magnetization of a system is stable and will remain in a certain state is of importance for probing the fundamental mechanism of magnetization reversal. Mono-domain magnetic particles become superparamagnetic, i.e., their time-averaged magnetization without a magnetic field is zero when their magnetic energy $K \times(4 / 3) \pi r^{3}$ is lower than about ten times the thermal energy $k_{\mathrm{B}} T$, with $r$ being the particle radius of a supposed spherical particle and $k_{\mathrm{B}}$ the Boltzmann constant (Leslie-Pelecky and Rieke 1996). At room temperature, $k_{\mathrm{B}} T=4.0 \times 10^{-21} \mathrm{~J}$, and one finds a maximum radius $r=6 \mathrm{~nm}$ for a superparamagnetic spherical particle of iron.

A typical magnetic nanoparticle consists of a magnetic core of diameter $\varnothing$, surrounded by a non-magnetic coating for selectively binding the biomaterial of interest (e.g., a specific cell, protein, or DNA sequence) (see Fig. 1a). Iron oxides, such as magnetite $\left(\mathrm{Fe}_{3} \mathrm{O}_{4}\right)$ or maghemite $\left(\gamma-\mathrm{Fe}_{2} \mathrm{O}_{3}\right)$ are more stable against oxidation, and are preferentially used as core material instead of iron. Typically, superparamagnetic particles of $\mathrm{Fe}_{3} \mathrm{O}_{4}$ (a)

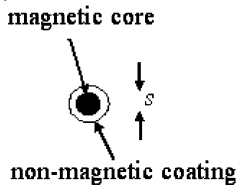

(b)

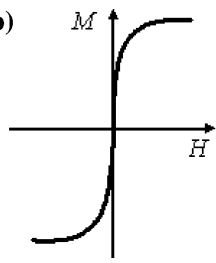

(c)

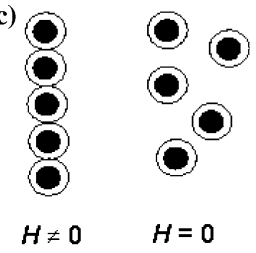

Fig. 1 a Schematic diagram of a spherical magnetic nanoparticle with internal core of diameter $s$, surrounded by a non-magnetic coating layer. b Schematic magnetization loop of an ensemble of superparamagnetic particles. c Schematic diagram of a nanoparticle superstructure in the presence of a magnetic field $H$. When the field is removed, the superstructure decomposes into single particles with diameters in the range $5-100 \mathrm{~nm}$ are used. The magnetization curve of an ensemble of such supermagnetic particles (see Fig. 1b) is hysteresis-free (at least, at not too high frequencies). This has important consequences for bio-analysis, as suspended superparamagnetic particles tagged to the biomaterial of interest can be removed from a matrix using a magnetic field, but they do not agglomerate (i.e., they stay suspended) after removal of the field (see Fig. 1c). Hence, it is very easy to switch on and off the magnetic interaction. Other advantages of using small nanoparticles are the minimum disturbance of the attached biomolecules (Hancock and Kemshead 1993) and a large surface-to-volume ratio for chemical binding.

Larger magnetic particles (typically $0.5-5 \mu \mathrm{m}$ in diameter) can have a single magnetic core or have a core composed of multiple more or less magnetically interacting nanoparticles in a non-magnetic matrix (see Fig. 2a). Such microparticles mostly have a multi-domain structure and are characterized by a hysteretic magnetization characteristic (see Fig. 2b). This means that, after the removal of the field, they keep a non-zero remnant magnetization $M_{\text {rem }}$, leading to magnetic bead clustering (see Fig. 2c). For example, spherical particles with radius $r=1.5 \mu \mathrm{m}$ with a saturation magnetization $\mu_{0} M_{\text {sat }}$ $=0.2 \mathrm{~T}$ and a remnant magnetization $\mu_{0} M_{\mathrm{rem}}=1 \mathrm{mT}$, will have a remnant magnetic moment $m_{\mathrm{rem}}=V \mu_{0} M$ rem $=(4 / 3) \pi r^{3} \mu_{0} M_{\text {rem }}=1.4 \times 10^{-20} \mathrm{Tm}^{3}$, with $\mu_{0}$ being the magnetic permeability in a vacuum, and $V$ the magnetic particle volume; two of such particles will have a maximum magnetic attraction energy $\left|U_{\text {max }}\right|=\frac{m_{\text {rem }}^{2}}{2 \pi \mu_{0}} \frac{1}{(2 r)^{3}}=9.2 \times 10^{-19} \mathrm{~J}$, much larger than the thermal energy, resulting in strong dipolar forces between the particles (Chikazumi 1964). As a consequence, when exposed to an external magnetic induction field, the magnetic microparticles acquire a magnetic dipole moment and coalesce, under the influence of the magnetic dipole interaction, into a supraparticle structure (SPS), consisting of chain-like "columnar" structures along the field direction. The exact shape of this SPS depends on the parameters, such as the particle concentration and the applied magnetic field. It is known, for example, that a constant and homogeneous magnetic field enables the (a)

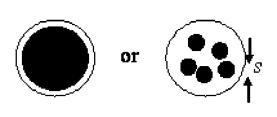

(b)

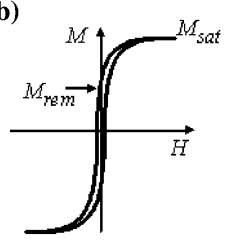

(c)

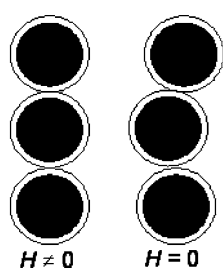

Fig. 2 a Schematic diagram of a spherical magnetic microparticle with a single internal magnetic core or consisting of multiple nanometer-sized cores of diameter $s$. b Schematic magnetization loop of an ensemble of ferromagnetic particles, showing magnetic hysteresis. $\mathbf{c}$ Schematic diagram of a microparticle superstructure in the presence of a magnetic field $H$. When the field is removed, the particles keep a remnant moment and the superstructure does not decompose 
generation of equally spaced arrays of columns of chains in a microchannel (Wang et al. 1994; Sandre et al. 1999; Hayes et al. 2001a).

To understand how a magnetic field can be used to transport and manipulate magnetic beads, it is important to recognize that a magnetic field gradient is required to exert a translation force - a uniform field gives rise to a torque, but no translational action. The magnetic force acting on a point-like magnetic dipole or "magnetic moment" $\mathbf{m}$ can be written as the derivative of the magnetic energy (Zborowski et al. 1999) ${ }^{2}$ :

$\mathbf{F}_{\mathbf{m}}=\frac{1}{\mu_{0}} \nabla(\mathbf{m} \cdot \mathbf{B}) \approx \frac{1}{\mu_{0}}(\mathbf{m} \cdot \nabla) \mathbf{B}$

The second part of the equation holds when the magnetic moment of the particle is not varying in space $(\nabla \cdot \mathbf{m}=0)$. Note that this is only a correct assumption when the moment is permanent, or the magnetic particle is in such a large field that its magnetization is completely saturated. As an example, for a constant moment $\mathbf{m}$ in the $x$ direction, $\mathbf{m} \cdot \nabla=m_{x} \frac{\partial}{\partial x}$, and a force will be exerted on the moment, provided that there is a magnetic field gradient in the $x$ direction (Chikazumi 1964). In the case of superparamagnetic nanoparticles in a nonmagnetic medium, one can write for the moment $\mathbf{m}=V \mu_{0} \mathbf{M}=V \mu_{0} \chi \mathbf{H}$, with $\mathbf{M}$ being the magnetization of the particle and $\chi$ is the magnetic susceptibility. Using the relation $\mathbf{B}=\mu_{0} \mathbf{H}$, Eq. 1 becomes:

$\mathbf{F}_{\mathbf{m}}=\frac{V \chi}{\mu_{0}}(\mathbf{B} \cdot \nabla) \mathbf{B}$

For example, when the magnetic particles are constrained to move in a microchannel in the $x$ direction, the $x$ component of the force becomes:

$\mathbf{F}_{\mathbf{m}, x}=\frac{V \chi}{\mu_{0}}\left(B_{x} \frac{\partial}{\partial x}+B_{y} \frac{\partial}{\partial y}+B_{z} \frac{\partial}{\partial z}\right) B_{x}$

This equation shows that both the magnitude of the magnetic field (for inducing a large moment) and the magnetic field gradient need to be large, to have a strong magnetic actuation force. However, one should be reminded that, if both the field and the moment have a spatial dependence, the first part of Eq. 1 needs to be considered and the force expression becomes more complicated.

Most magnetic actuation is done using permanent magnets, rather than with coils. A permanent magnet is typically characterized by a magnetic induction $B_{\mathrm{m}}=0.5-1 \mathrm{~T}$ and a field gradient $\nabla B \approx B_{\mathrm{m}} / w$, with $w$ being the typical geometrical dimension of the permanent magnet. For a cylindrical permanent magnet with a diameter $\varnothing=5 \mathrm{~mm}$, one induces on a spherical particle

\footnotetext{
${ }^{2}$ Here, we use the definition of force where the moment has units of $\mathrm{T} \mathrm{m}^{3}$, as introduced in the book of Chikazumi (1964). An alternative dimension in literature for the moment is $\mathrm{A} \mathrm{m}^{2}$, but, in this case, the factor $\mu_{0}$ in the denominator of Eq. 1 needs to be replaced by 1 .
}

with radius $r=500 \mathrm{~nm}$ and $\chi=1$, a magnetic moment $m=2.6 \times 10^{-19} \mathrm{Tm}^{3}$, resulting in a magnetic force of about $40 \mathrm{pN}$. For a current-fed coil, the generated field is much smaller: a flat millimeter-size coil with ten windings and a current of $0.5-1$ A typically generates a magnetic induction of $1-10 \mathrm{mT}$, at least 100 times smaller than the permanent magnet. Consequently, the gradient is also a factor of 100 lower, so the force of Eq. 2 is a factor of $10^{4}$ larger when using a permanent magnet rather than a simple coil.

In many applications, a magnetically labeled material is separated from a liquid solution by passing the fluid mixture through a region where there is a magnetic field gradient that can immobilize the tagged material via magnetic forces. For in vivo applications, magnetic particles can be transported by the blood flow and locally retained by the application of an external magnet. In other applications, there is a magnetic translational driving force and the liquid solution is static. In all examples, the magnetic force needs to be larger than the hydrodynamic drag force acting on the magnetic particle. The hydrodynamic drag force is a consequence of the velocity difference between the magnetic particle and the liquid $\Delta \mathbf{v}$ and, for a spherical particle with radius $r$, is given by (White 1999):

$\mathbf{F}_{\mathrm{d}}=6 \pi \eta r \Delta \mathbf{v}$

where $\eta$ is the viscosity of the medium surrounding the particle (for water, $\eta=8.9 \times 10^{-4} \mathrm{~N} \mathrm{~s} / \mathrm{m}^{2}$ ). Equalizing Eqs. 2 and 4 permits us to determine the maximum flow rate that a particle can withstand when exposed to a magnetic immobilization force, or the maximum particle flow rate that can be generated by a magnetic force in a surrounding static liquid:

$\Delta \mathbf{v}=\frac{2 r^{2} \chi(\mathbf{B} \cdot \nabla) \mathbf{B}}{9 \mu_{0} \eta} \equiv \frac{1}{\mu_{0}} \xi(\mathbf{B} \cdot \nabla) \mathbf{B}$

with:

$\xi \equiv \frac{2 r^{2} \chi}{9 \eta}=\frac{V \chi}{6 \pi r \eta}$

being the "magnetophoretic mobility" of the particle, which is a parameter describing how magnetically manipulable the particle is. One notes that, in general, the quantity $(\mathbf{B} \cdot \nabla)$ B can be a strongly varying function in space, which implies a similar spatial variation for $\Delta \mathbf{v}$. Moreover, in the case of strong spatial variations of the field, the magnetic moment of the particle will not be constant when moving in space and the general form (first part) of Eq. 1 needs to be considered, giving rise to a more complicated expression for the magnetic force.

\section{Magnetic fluids}

Magnetic bead solutions or magnetic fluids are stable dispersions of magnetic beads or encapsulated magnetic 
particles in an organic or aqueous carrier medium. Specific applications of magnetic beads require a set of necessary particle properties. Requirements of the particle matrix, such as biocompatibility, biodegradability, and stability in different media, must be combined with a uniform size distribution and a correct size range and shape. Physical properties, like the iron oxide content, determine the magnetic behavior of the beads and must be associated with suitable bead surface modifications (hydrophilicity versus hydrophobicity, surface functionality, etc.) in order to allow covalent bonding or simple unspecific adsorption of biomolecules (proteins, antibodies, nucleic acids). Usually, the magnetic beads are tailor made for a specific final application.

\subsection{Synthesis}

Reviews of the synthesis of inorganic nanoparticles in the liquid phase (not limited to magnetic materials) were presented by Grieve et al. (2000), Trindade et al. (2001), and Murray et al. (2000), while a review of the synthesis of such particles from the vapor phase was presented by Swihart (2003). The synthesis and applications of (nonmagnetic) polymer microparticles were reviewed by Kawaguchi (2000). The articles of Landfester and Ramirez (2003), Bergemann et al. (1999), and Gruttner et al. (2001) present specific short review sections on the synthesis and chemical modifications of magnetic beads. The synthesis of magnetic beads is also a well covered subject in the patent literature.

In early publications, magnetic bead solutions were produced by grinding magnetite $\left(\mathrm{Fe}_{3} \mathrm{O}_{4}\right)$ with long-chain hydrocarbons and a grinding agent (Papell 1965). Later magnetic fluids were produced by precipitating an aqueous $\mathrm{Fe}^{3+} / \mathrm{Fe}^{2+}$ solution with a base, coating these particles with an adsorbed layer of oleic acid and then dispersing them in a non-aqueous fluid (Reimers and Khalfalla 1974). Both types of process result in very small magnetic particles with a surfactant coating in a non-aqueous liquid carrier in which the hydrophobic magnetite particles are dispersed. However, a lot of applications of magnetic beads rely on water as the continuous phase.

A water-based magnetic fluid was realized by the conversion of iron products to magnetic iron oxide in an aqueous medium under controlled $\mathrm{pH}$ conditions (Kelley 1977). Polymer-coated magnetic beads can be produced by in situ precipitation of magnetic materials in the presence of a polymer. In this way, magnetic beads surrounded by a hydrophilic polymer shell have been made, choosing for the polymer the water-soluble dextran (Molday 1984), poly-(ethylene imine) (Rembaum 1981), poly-(vinyl alcohol) (Lee et al. 1996), poly-(ethylene glycol) (Suzuki et al. 1995), etc. Figure 3a is a transmission electron microscopy (TEM) photograph of dextran nanoparticles; note that the dextran is not visible in the TEM picture and that the particles have an irregular (non-spherical) shape. Figure $3 \mathrm{~b}$ is a TEM photograph of more regular polystyrene particles with encapsulated magnetite nanoparticles.

Single basic types of magnetic beads have their individual advantages and disadvantages. Polystyrenecoated magnetic particles are known for their excellent size distribution and spherical shape (Dynal; Singer 1987). However, their hydrophobic surface results in a high amount of unspecific protein and antibody binding on the particle surface, so it needs to be modified chemically. Magnetic silica particles are very efficient in adsorbing proteins and DNA on their surface, but are hardly available with a small size distribution and an ideal spherical shape (Kleiber et al. 2001; Undisclosed_inventors 2001). Magnetic polysaccharide particles are important for many in vivo applications. They combine biocompatibility with availability in a size range below $300 \mathrm{~nm}$ (Miltenyi et al. 1990; Gruttner and Teller 1999), but the particles are irregular in shape and the soft particle matrix causes them to be sensitive to mechanical stress. Also, magnetic poly-(lactic acid) particles play an important role in in vivo applications (Hafeli et al. 1994; Hafeli and Pauer 1999); they are biodegradable and their degradation time in the blood can be adjusted by their molecular weight and exact chemical composition. However, because of their
Fig. 3 a Transmission electron microscopy (TEM) photograph of dextran nanoparticles; the dextran coating is not visible in the TEM picture and the particles have an irregular shape (reprinted from Gruttner and Teller 1999, with permission from Elsevier). b More regular polystyrene particles with encapsulated magnetite nanoparticles (reprinted from Landfester and Ramirez 2003, with permission from The Institute of Physics Publishing Ltd.)
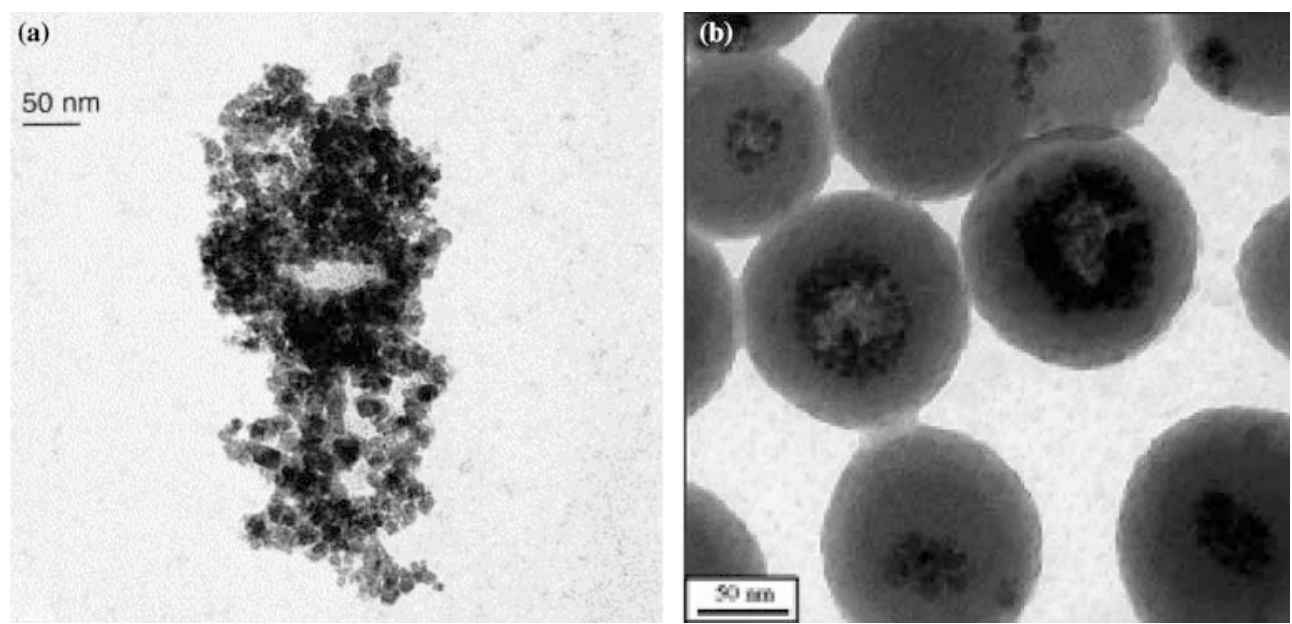
hydrophobic surfaces, these particles stick to plastic surfaces like pipette tips and plastic recipients, resulting in problems with particle handling and analytical errors.

\subsection{Applications in biomedicine}

The use of magnetic bead solutions for biomedical applications, but excluding microfluidic applications, has been reviewed several times (Roger et al. 1999; Safarik and Safarikova 2002; Shinkai 2002; Pankhurst et al. 2003; Bauer et al. 2004). The fact that a magnetic field gradient can be used to either remotely position or selectively filter biological materials attached to a magnetic bead has led to a number of obvious applications. The latter broadly fall into two categories: those involving the use in vivo and those involving the use of magnetic beads in vitro. An important in vivo application is using the magnetic beads to produce a distortion in the magnetic field at a given site under examination via magnetic resonance imaging (MRI). The presence of magnetic beads strongly alters the contrast of cells, enabling visualization of hitherto impossible objects. Another in vivo application lies in the production of controlled heating: a high-frequency magnetic field can be used to selectively warm a given area and destroy malignant tissue by a phenomenon known as hyperthermia. Magnetic beads can also play a role in drug targeting, as they can be vectors for carrying the drug and can be magnetically guided to the desired location. For in vitro applications, the possibility of the specific labeling of cells or biomolecules with magnetic microbeads has lead to advanced developments in cell manipulation and biomolecule separation, DNA sequencing, and biomolecule selection and purification.

\subsubsection{MRI contrast enhancement}

In MRI, image contrast is a result of the different signal intensities that each tissue produces in response to a particular sequence of applied radio frequency pulses. This response depends on the proton density and magnetic relaxation times of the tissue. Target-specific superparamagnetic particles can serve as a dramatic source of contrast and have become an indispensable tool for the non-invasive study of biological processes with MRI. Superparamagnetic particles change the local rate at which protons decay from their excited state to the ground state. When biocompatible dextran- or starch-coated particles selectively bind to healthy or diseased cells, they generate a local magnetic dipole in the large field of the MRI setup which, by a change in the local proton relaxation time, is at the basis of the imaging contrast between the different types of cells (Lawaczeck et al. 1997; Kim et al. 2001a, 2001b, 2003). There is also a size effect: for example, nanoparticles with diameters of ca $30 \mathrm{~nm}$ or more are rapidly collected by the liver and spleen, while particles with sizes of ca $10 \mathrm{~nm}$ or less are not so easily recognized. The smaller particles, therefore, have a longer half-life time in the blood and are collected by certain types of cells in the lymph nodes and the bone marrow (Ruehm et al. 2001). As tumor cells do not have the effective type of cells to take up the nanoparticles, MRI assisted in imaging malignant lymph nodes or liver tumors (Semelka and Helmberger 2001).

\subsubsection{Hyperthermia}

Hyperthermia is one of the most promising approaches in cancer therapy: it consists in heating and destroying the target tissue to temperatures between $42^{\circ} \mathrm{C}$ and $46^{\circ} \mathrm{C}$. Various methods are employed in hyperthermia, such as the use of hot water, capacitive heating, and inductive heating of malignant cells (Cavalier et al. 1967; Stauffer et al. 1984; Lin and Wang 1987; Ikeda et al. 1994). The problem with hyperthermia is the difficulty of local heating of only the tumor region until the required temperature is reached, without damaging the surrounding normal tissue. Magnetic particle hyperthermia is appealing because it offers a way to ensure that only the intended target tissue is heated. The concept is based on the principle that a magnetic particle can generate heat by hysteresis loss when placed in a high-frequency $(\sim 1 \mathrm{MHz})$ magnetic field (Stauffer et al. 1984). Intracellular hyperthermia using dextran nanoparticles dates from 1979 (Gordon et al. 1979). The principle of heating with superparamagnetic particles (that show no magnetic hysteresis at low frequencies) by an AC field has been reviewed by Rosensweig (2002): the dissipation results from the orientational relaxation of the particles having thermal fluctuations in a viscous medium (Hergt et al. 1998). A number of studies have demonstrated the therapeutic efficacy of this form of treatment in animal models (for a review, see, for example, Moroz et al. (2002)), but the application of this technology to human patients is just starting (Jordan et al. 2001).

\subsubsection{Drug targeting}

A disadvantage of most chemotherapies is that they are relatively non-specific and induce secondary effects in the healthy tissue. In magnetically targeted therapy, a cytotoxic drug is attached to biocompatible magnetic carriers that are injected into the patient via the circulatory system. When the particles have entered the bloodstream, external high-gradient magnetic fields are used to concentrate the complex at a specific target site within the body. Once the drug carrier is concentrated at the target, the drug can be released either via enzymatic activity or changes in physiological conditions, such as $\mathrm{pH}$ or temperature (Alexiou 2001), and be taken up by the tumor cells. An overview of the clinical applications of magnetic drug targeting was given by Lubbe et al. (2001). Larger particles with dimension $>\mu \mathrm{m}$, comprising agglomerates of superparamagnetic particles, were shown to be more effective in withstanding flow dynamics within the circulatory system. In most cases, the magnetic field gradient is generated by a strong permanent magnet, such as 
$\mathrm{NdFeB}$, fixed outside the body on the target site. Limitations of the technique are: (1) the possibility of embolization of blood vessels in the target region due to the accumulation of magnetic carriers; (2) difficulties in scaling up from animal models due to the larger distances between the target site and the magnet; (3) the fact that, once the drug is released, it is no longer attracted to the magnetic field; and (4) toxic responses to the magnetic carriers (Pankhurst et al. 2003).

\subsubsection{Magnetic separation}

Separation of magnetic-bead-labeled biomolecules or cells from a liquid solution is a well documented and widely practiced application today, and is extremely important in process engineering. Many types of magnetic particles have been developed for use in separation processes, including purification processes and immunoassays (Dunnill and Lilly 1974; Mosbach and Andersson 1977; Kondo et al. 1994; Koneracka et al. 2002; Kourilov and Steinitz 2002). Automatic DNA/RNA purification systems based on magnetic separation are commercially available and magnetic cell sorting has been developed for cellular therapy applications.

Magnetic separator design can be as simple as the application and removal of a permanent magnet to the wall of a test tube to cause aggregation, followed by removal of the supernatant. However, it is preferable to increase the separator efficiency by producing regions with a high magnetic field gradient to capture the magnetic nanoparticles as they float or flow by in the carrier medium. Figure $4 \mathrm{a}$ is a schematic diagram of the socalled quadrupole magnet configuration, where four magnets are arranged in order to induce a maximum magnetic field gradient at the outer side of a liquid carrying tube, inserted into the free space between the magnets (Hatch and Stelter 2001). Besides this, other permanent magnet configurations have been proposed for separation (Haik et al. 1999; Todd et al. 2001; Nedelcu and Watson 2002; Hoffmann and Franzreb 2004). Figure $4 \mathrm{~b}$ is a calculation of the magnitude of the magnetic force (using Eq. 2) inside the quadrupole magnet assembly. Williams et al. (1999) described how such a quadrupole magnet can be combined with an annular
Fig. 4 a Schematic diagram of a classical quadrupole magnet configuration and the corresponding magnetic flux lines. b Calculation, using Eq. 2, of the force density in the bore of the quadrupole magnet. c A cylindrical capillary is inserted into the cross-section of a quadrupole magnetic cell sorter, and a cell separation sample is fed in at $a^{\prime}$, and carrier fluid at $b^{\prime}$. The inlet splitting surface (ISS) corresponds to the inner broken lines and the outlet splitting surface (OSS) to the outer broken lines. Sorted fractions are collected at outlets $a$ and $b$ (Figs. a and b reprinted from Hatch and Stelter 2001, with permission from Elsevier; Fig. c reprinted from Williams et al. 1999, with permission from the American Chemical Society) (a)

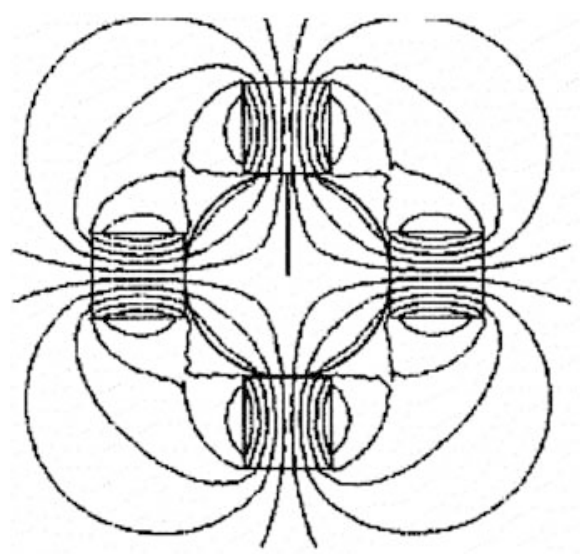

(b)

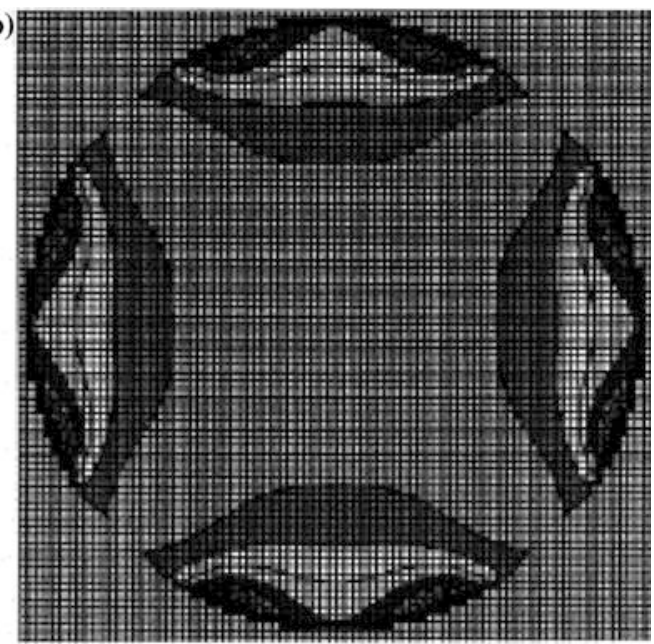

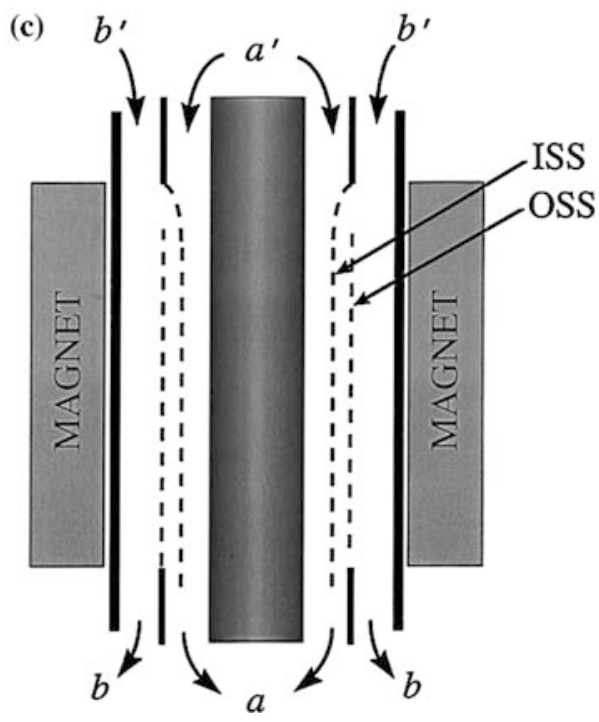


fluidic circuit that is schematically shown in Figure 4c. The separation takes place within a laminar flow of a carrier fluid along a thin annular channel. A field gradient is imposed across the thin dimension of the channel, perpendicular to the direction of the flow. The sample mixture is arranged to enter the system close to one of the channel walls and, as the sample is carried along the channel by the flow of the fluid, those components that interact more strongly with the field gradient are carried transversely across the channel thickness. A division of the flow at the channel outlet using a stream splitter completes the separation into fractions. The radial particle separation velocity is induced by the field gradient and can easily be calculated using Eq. 5. Of course, the fluid transport velocity should not exceed a maximum limit to enable a correct separation over the magnetic field gradient region. Using such a quadrupole magnetic sorter, magnetic cell separation was shown to be a function of the antibody binding capacity, which is related to the number of magnetic bead labeling sites of a single cell (McCloskey et al. 2003a, 2003b). An alternative way to obtain an efficient magnetic separation is to loosely pack the flow column with a magnetizable matrix of thin wires or beads (Rheinlander et al. 2000; Ebner and Ritter 2001), but such method can suffer from problems of desorption of the magnetic beads from the matrix after the removal of the field.

The magnetic separation systems discussed so far have a macroscopic dimension from the magnet point of view, in contrast to the microfluidic separation systems proposed in the next section. However, from a liquid transport point of view, they may be already called "microfluidic," as their functioning is based on the presence of laminar flow patterns, like found in microfluidic systems.

\subsubsection{Cell biophysics}

For applications like MRI imaging, hyperthermia and separation, specific binding to, or uptake by, a certain cell type is at the basis of the magnetically induced contrast. Superparamagnetic particle uptake kinetics of living cells and toxicity aspects of such uptake has been discussed by several authors (Wilhelm et al. 2002; Tiwari et al. 2003). Also, the incorporation of magnetic beads into cells has provided a new tool to measure cytoskeleton-associated cell functions (Valberg and Butler 1987; Wang et al. 1993) and ferromagnetic microparticles acted as inert tracers for cell function monitoring. For example, within macrophages, the particles are stable over weeks and months. Intracellular transport of the particles caused stochastic disorientations of the dipole particles, resulting in a decay of the magnetic cell field that can be measured by a sensitive magnetic field sensor. Magnetic particle twisting was used to investigate the mechanical integrity and visco-elastic properties of the cytoskeleton (Moller et al. 2003) and, in combination with optical tweezers, superparamagnetic beads were at the basis of force and torque measurement systems on single cells (Romano et al. 2003). Recently, cell-bound magnetic microparticles, subjected to $0.1 \mathrm{~ms}$ magnetic field pulses, have been used to destruct the targeted cells by penetration of the beads into the cells or by rupturing the cells with the beads (Ogiue-Ikeda et al. 2003), a nonthermal process which is different from hyperthermia.

\section{Magnetic bead handling microsystems}

\subsection{Magnetic beads as solid phases in heterogeneous assays}

As explained in the introduction, fluidic systems of reduced dimension have the advantage of reduced reagent consumption and waste generation, fast reaction times, and a large surface-to-volume ratio, offering an intrinsic compatibility between the use of a microfluidic system and surface-based assays. Immuno-assays are probably the most important analysis method for biological molecules, as the molecular recognition reaction provides high selectivity and chemical sensitivity. Although conventional microtiter plate assays continue to play an important role, enzyme assays, DNA binding, and competitive immuno-assays have been performed on microdevices (Koutny et al. 1996; Chiem and Harrison 1997; Hadd et al. 1997). The three cited experiments were homogeneous immuno-assays in which both the antigen and the antibody molecules are simultaneously introduced in a solution, complexation takes place, and an electrophoretic separation and detection follows. Separation was possible because electrophoretic migration times between complexed and free-form antigens or antibodies were sufficiently different.

Heterogenous assays, where reactions occur both in solution and in a solid phase, offer the advantage of easy separation of chemical complexes from reactants. Biomolecule immobilization on a solid phase, formed by the surface of microparticles or nanoparticles, evidently results in a small-volume and localized assay (Rashkovetsky et al. 1997; Sato et al. 2000). Such a solid phase provides a high surface-to-volume ratio, reducing diffusion times during the microfluidic procedures, and increasing the density of binding sites, which is beneficial for a high detection signal and sensitivity. In addition, such an assay allows for a rapid regeneration and exchange of the solid support when needed. Magnetic beads locally immobilized by a magnetic field clearly offer these advantages ${ }^{3}$.

Magnetic beads have been combined with commercial capillary electrophoresis (CE) instrumentation for performing enzymatic and inhibition assays, as well as for the analysis of biological molecules, such as antigens (Rashkovetsky et al. 1997). For example, a small quantity of

\footnotetext{
${ }^{3}$ The advantages related to the use of magnetic beads in microfluidic systems apply equally well to the more "macroscopic" microtiter plate and test tube formats.
} 
polystyrene magnetic microbeads containing immobilized biomolecules was injected into a neutral hydrophiliccoated fused-silica capillary. The short plug $(2-3 \mathrm{~mm})$ of beads was held fixed by a magnet placed in the cartridge of the $\mathrm{CE}$ system. The microfluidic procedure involved antigen capture from the sample solution to the beads, rinsing, elution, and electrophoretic separation of the sample. The beads could be replaced after each run, eliminating the need to regenerate the solid support.

In another study, a small-volume heterogeneous immuno-assay was demonstrated using both classical fused-silica capillaries and glass microfluidic chips (Hayes et al. 2001b). Figure 5 is a schematic diagram of the experimental configuration. The system demonstrated the presence of the parathyroid hormone at concentrations of $1 \mu \mathrm{g} / \mathrm{l}$, using a sandwich assay, which was based on the trapping or capture of the analyte by one antibody and the detection by another (fluorescent) antibody. Also, a calibration curve was generated at clinically relevant levels $(2-250 \mu \mathrm{g} / 1)$ for the detection of interleukin-5, an important cytokine for asthma, immune response cascades, and cancer.

Dynamic DNA hybridization was demonstrated by immobilizing DNA targets on magnetic beads via streptavidin-biotin conjugation or base pairing between oligonucleotide residues (Fan et al. 1999). The DNA/ bead complex was introduced into the device, after which, hybridization took place with a complimentary probe. The hybridized probe was then removed by heat denaturation to allow the DNA sample to be interrogated again by another probe with a different sequence of interest. Demonstration of specific hybridization reactions in an array format was achieved using four synthesized DNA targets and five probes in sequence, indicating the potential of this approach for gene expression analysis. In another study, mRNA was isolated from total RNA in a flowing stream within a microchip using polystyrene magnetic microbeads labeled with a poly-thymine tail for mRNA capture (Jiang and Harrison 2000). A few nanograms of mRNA could be captured per microgram of total RNA.

Choi et al. (2002) reported on the development and characterization of an integrated microfluidic biochemical detection system for fast and low-volume immunoassays using magnetic beads (Choi et al. 2002). First, antibody-labeled polystyrene magnetic beads were separated from a liquid solution by a set of planar coils. While holding the antibody-coated beads, antigens were injected into the channel. Only target antigens were immobilized, and, thus, separated onto the magnetic bead surface due to the specific antibody/antigen reaction. Detection of the target antigen was based on an electrochemical enzymatic reaction in a sandwich immuno-assay. In another study, a fully integrated biochip cartridge that consists of microfluidic mixers, valves, pumps, channels, chambers, heaters, and DNA microarray sensors was developed to perform DNA analysis of complex biological sample solutions (Liu et al. 2004). Sample preparation (including magnetic-bead-based cell capture, cell pre-concentration and purification, and cell lysis), polymerase chain reaction (PCR), DNA hybridization, and electrochemical detection were performed in this fully automated miniature device. Pathogenic bacteria detection from milliliter whole blood samples and single-nucleotide polymorphism analysis was demonstrated directly from diluted blood.

\subsection{Magnetic beads as labels for detection}

A common approach to detecting biological molecules is to attach to the target molecule a label that produces an
Fig. 5 Schematic

representation of a flow-based micro-immuno-assay system.

The magnetic beads are collected from a dilute solution near a rare earth magnet to form a packed bed within a microfluidic channel. Reagents are introduced into the packed bed to perform standard immuno-assays and the bed is imaged with an epifluorescence microscope with laser-induced excitation. (Reprinted from Hayes et al. 2001, with permission from the American Chemical Society)
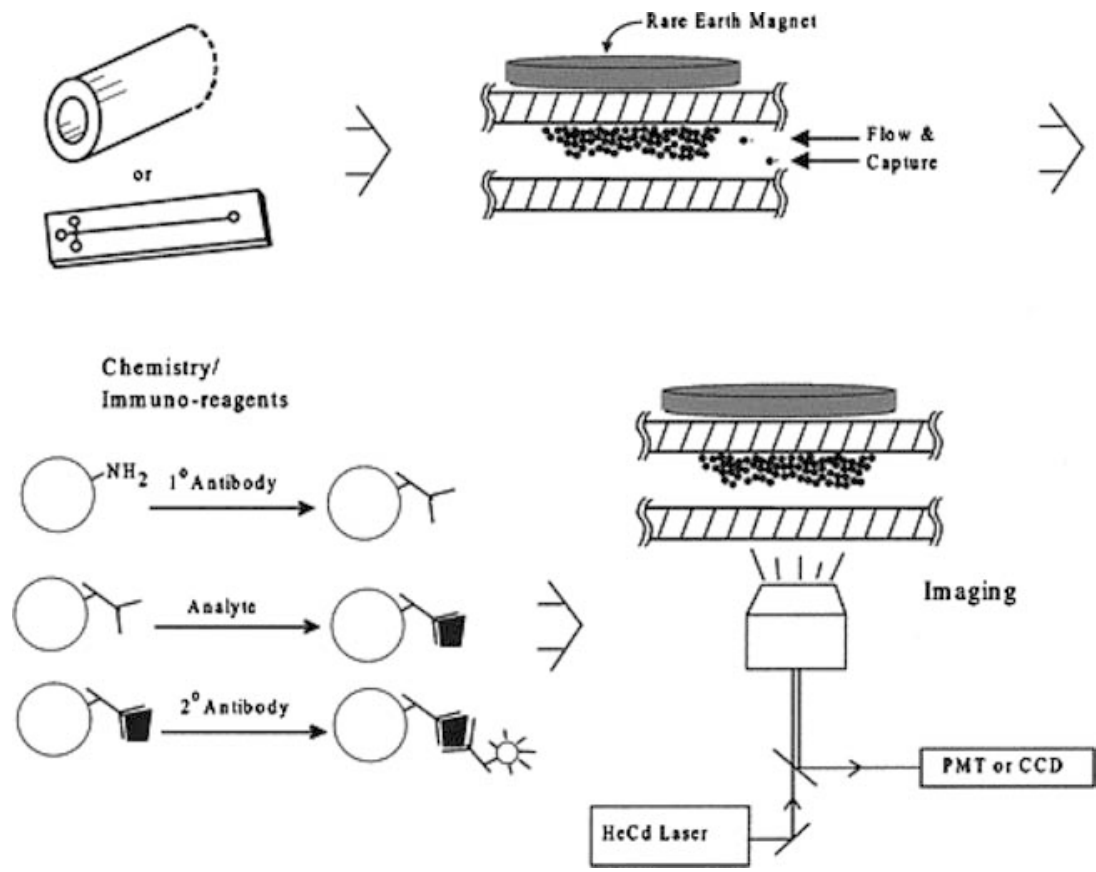
externally observable signal. Traditionally, this is accomplished using biomolecular recognition between the target molecule and a specific receptor (e.g., an antibody) that is tagged with the label. The label may be a radioisotope, enzyme, fluorescent molecule, or charged molecule, for example. Recently, magnetic beads have also been used as labels for biosensing. Magnetic labels have several advantages over other labels. The magnetic properties of the beads are stable over time, in particular, because the magnetism is not affected by reagent chemistry or subject to photo-bleaching (a problem with fluorescent labeling). There is also no significant magnetic background present in a biological sample and magnetic fields are not screened by aqueous reagents or biomaterials. In addition, magnetism may be used to remotely manipulate the magnetic particles. Finally, a number of very sensitive magnetic field detection devices have been developed during recent years, such as giant magnetoresistance (GMR) (Baibich et al. 1988) and spin-valve (Dieny et al. 1991; Freitas et al. 2000) magnetic sensors that enable the measurement of extremely weak magnetic fields, for example, the magnetic field generated by the magnetization of a single magnetic microbead. A basic GMR or spin-valve device consists of a pair of magnetic films separated by a non-magnetic conducting layer (Hartmann 1999). When an external magnetic field rotates the magnetizations of the magnetic layers towards alignment, spin-dependent electron scattering is reduced at the interfaces within the device, decreasing its electrical resistance. GMR sensors can be microscopic in size and are sensitive to the presence of micron, and smaller, size magnetic particles when in close proximity. Besides GMR sensors, measurements of single magnetic beads have been demonstrated using miniaturized silicon Hall sensors (Besse et al. 2002) and planar Hall effect sensors based on permalloy thin films (Ejsing et al. 2004).

A research group at the Naval Research Laboratory, Washington DC (Baselt et al. 1998; Edelstein et al. 2000; Miller et al. 2001; Rife et al. 2003), followed by others (Coehoorn and Prins 2003; Graham et al. 2003), have developed a microsystem for the capture and detection of micron-sized, paramagnetic beads on a chip containing an array of GMR sensors, the so-called bead array counter (BARC). The BARC is based on a sandwich assay, where the target molecule is bound to an immobilized probe on the GMR sensor, after which, the magnetic label is bound to the target using specific ligand-receptor interactions. A schematic diagram of a test experiment is shown in Fig. 6: thiolated DNA probes are patterned onto a gold layer directly above the GMR sensors on the BARC chip. To prevent non-specific adhesion of sample DNA and of the magnetic beads to the unmodified areas of the gold surface, the arrayed surfaces are passivated with thiolated polyethylene glycol (PEG). Biotinylated sample DNA is then added, which hybridizes with the DNA probes on the surface when the complementary sequence is present, after which, the unbound sample DNA is washed away. Then, streptavidin-coated magnetic beads are injected over the chip surface, binding to biotinylated sample DNA hybridized on the BARC chip. A magnetic field gradient can be applied to attract magnetic beads to the chip or to selectively pull off only those beads not bound to the surface by specific binding. The field can be generated by external permanent magnets or electromagnets (Lee et al. 2000), electromagnets integrated with the chip (Edelstein et al. 2000), or by current-carrying lines positioned close to the chip (Lagae et al. 2002). Bound beads are detected by the GMR sensors by applying a uniform magnetic field perpendicular to the substrate, imposing a magnetic moment to the superparamagnetic beads. This induced moment generates an in-plane magnetic field that is measured by the GMR sensor. Applying the uniform field normal to the plane of the GMR sensor rather than in-plane has the advantage that, due to demagnetization effects, a much larger magnetizing field can be applied to the beads without saturating the sensor (Tondra et al. 2000). Figure 7 shows that single $2.8-\mu \mathrm{m}$ diameter magnetic beads can be detected using $80 \times 5-\mu \mathrm{m}^{2}$ GMR sensor strips. The graphs show the voltage signals, corresponding to the resistance change, from 16 of the 64 sensor elements, with and without adsorbed beads. The signal due to a single bead varies from strip to strip, depending on whether the bead is directly on top of the sensor (elements 2 and 15), not present (11 and 14), or near the edge of the sensor (all others). The noise level is determined by measuring the signal before the beads are injected and after they are washed off.

The GMR sensor sensitivity increases with decreasing surface area of the sensor; however, the chemical sensitivity, or the number of analyte molecules that can

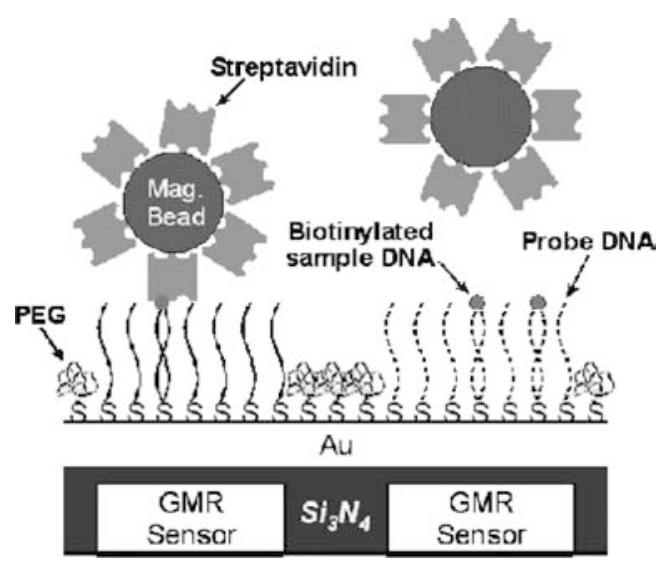

Fig. 6 Schematic diagram of the BARC chip surface chemistry and hybridization assay. Thiolated DNA probes are patterned onto a gold layer directly above the GMR sensors on the BARC chip. Biotinylated sample DNA is then added, which hybridizes with the DNA probes on the surface when the complementary sequence is present. The unbound sample DNA is washed away. Streptavidincoated magnetic beads are injected over the chip surface, binding to biotinylated sample DNA hybridized on the BARC chip. Beads that are not specifically bound are removed by applying a magnetic field. Bound beads are detected by the GMR sensors (reprinted from Edelstein et al. 2000, with permission from Elsevier) 
hybridize to the surface, increases with increasing surface area. Theoretical modeling showed that a GMR sensor can detect a single superparamagnetic bead of any size (Tondra et al. 2000; Li et al. 2003; Li and Wang 2003), as long as all system dimensions (bead size, sensor size, distance between bead and sensor) scale down proportionally. When the sensor size can be of the size of the bead, it should be possible to detect beads with a radius down to $100 \mathrm{~nm}$ or smaller.

Most bead-sensing experiments today have been done with 2.8- $\mu \mathrm{m}$ size beads composed of magnetic $\gamma-\mathrm{Fe}_{2} \mathrm{O}_{3}$ and $\mathrm{Fe}_{3} \mathrm{O}_{4}$ nanoparticles dispersed in a polystyrene matrix with an average magnetic content of 17 weight $\%$ (Dynal). To maximize the sensor response, the magnetic beads should have a magnetization as high as possible, and yet, remain non-remnant to avoid clustering when suspended in solution. With the goal of achieving larger signals from the magnetic labels, soft ferromagnetic beads with $100 \%$ magnetic content and a much higher saturation magnetization have been developed (Miller et al. 2001). $\mathrm{NiFe}$ beads of $1-\mu \mathrm{m}$ diameter showed a susceptibility of $\sim 3$, the maximum obtainable value for a uniformly magnetized sphere. Because of this property, smaller solid ferromagnetic beads could effectively be used as biomagnetic labels, which would also increase the dynamic range of biosensor assays by allowing more labels per unit area.

\subsection{Separation of magnetic beads}

Magnetic separation, as discussed in a previous section, is already an important application of magnetic beads.

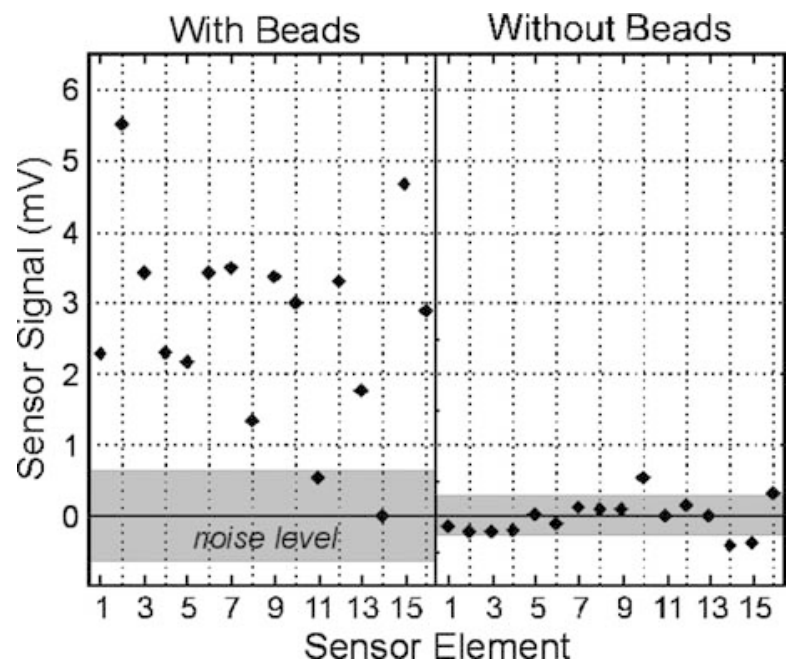

Fig. 7 Detection of magnetic beads with the BARC chip. The graphs show the signals from 16 of the 64 sensor elements, with and without adsorbed beads. The signal due to a single bead varies from element to element, depending on whether the bead is directly on top of the sensor (elements 2 and 15), not present (11 and 14), or near the edge of the sensor (all others). The noise level is determined by measuring the signal before the beads are injected and after they are washed off (reprinted from Edelstein et al. 2000, with permission from Elsevier)
While the magnetic separation systems discussed so far have a macroscopic dimension from the magnet point of view, some of them (Williams et al. 1999; Zborowski et al. 1999) might be already called "microfluidic," as their functioning was based on the presence of laminar flow in a fluidic channel. Also, microfluidic channels made in silicon wafers have been combined with closely positioned permanent magnets to separate magnetic from non-magnetic particles into different output channels (Blankenstein and Larsen 1998). In many of the "macroscopic" applications, high magnetic field gradients were achieved by using the high background field gradient of a permanent magnet or an electromagnet in combination with a filter of closely packed steel wool. Most of the latter approaches, although successful, have the drawback that close monitoring of the separation process is difficult by the nature of the filter geometry.

A truly integrated magnetic bead sorter combines micromagnetics with microfluidics and is characterized by a magnetic environment where particles with distinct magnetic properties may be manipulated differently to other particles on the length scale of the microfluidic circuit. The group of Ahn reported several of such integrated bead separation systems (Ahn et al. 1996; Choi et al. 2000, 2001). Such devices can be used, for example, for miniaturized cell sorting, where the magnetic beads are used as a magnetic "label" for actuation, or in a miniaturized bio-reaction system, where the magnetic beads play the role of the carrier substrate in an assay (Choi et al. 2000, 2002). Figure 8 is a schematic diagram of a sandwich immuno-assay that uses magnetic separation. Antibody-coated beads are introduced on the electromagnet (Fig. 8a) and are separated by applying magnetic fields. While holding the antibodycoated beads (Fig. 8b), antigens are injected into the channel (Fig. 8c). Only target antigens are immobilized and, thus, separated onto the magnetic bead surface due to antibody/antigen interaction (Fig. 8d). Other antigens get washed out with the flow. In a subsequent optional step, labeled secondary antibodies can be introduced and incubated with the immobilized antigens, after which, the chamber is rinsed to remove all unbound secondary antibodies. Finally, the magnetic beads are released to a sensing chamber of the bio-assay (Fig. 8e) and the bioseparator is ready for the next sample. Alternatively, sensing may be based on the optical detection of an electro-chemiluminescent label attached on the magnetic beads, and can be done directly in the separation chamber (Choi et al. 2002). Figure 9 is an experimental separation of $1-\mu \mathrm{m}$ diameter magnetic beads from a liquid flow. The separation is realized within a microfluidic channel in a silicon wafer that is integrated on top of a set of two planar electromagnets. Each of the electromagnets has a width of $50 \mu \mathrm{m}$ and consists of a spiral $\mathrm{Cu}$ winding, electroplated in a photoresist mold and integrated with a soft magnetic permalloy yoke and backing plate. The latter serves to enhance the generated field by about a factor of two with 


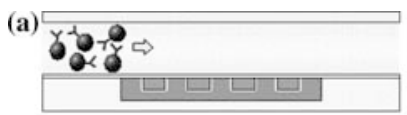

(b)

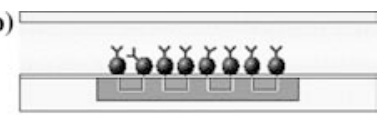

(c)

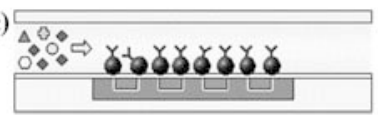

(d)

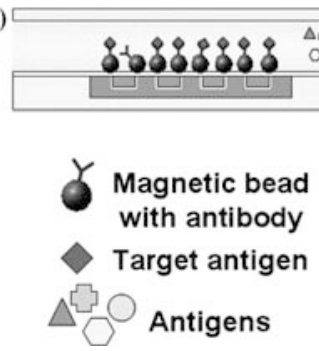

Fig. 8a-e Conceptual illustration of the use of a micromachined magnetic separator for bio-sampling. a Injection of the magnetic beads. b Separation and holding of the beads by a planar electromagnet. c Flowing through the sample solution. d Immobilization of target antigen. e Release of the labeled magnetic beads. (Reprinted from Choi et al. 2000, with permission from Elsevier)

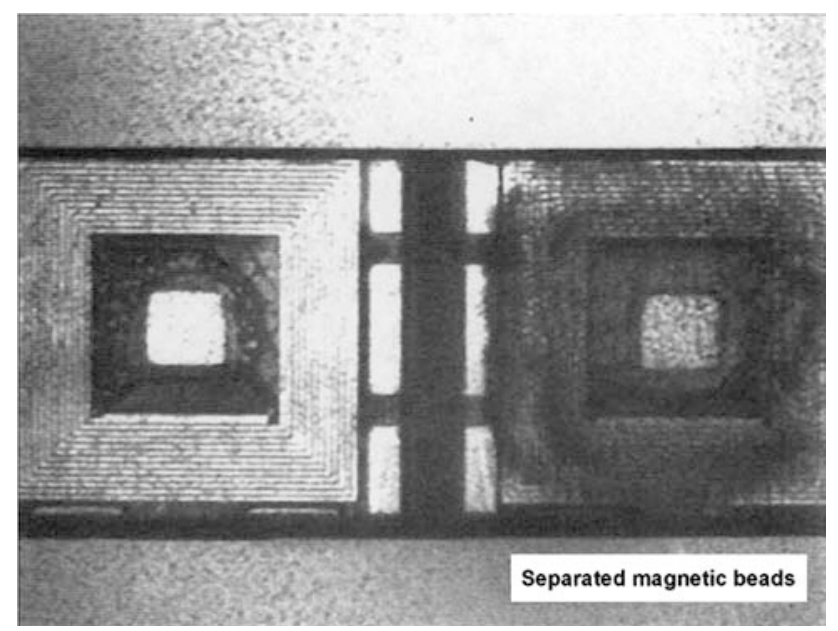

Fig. 9 Separation of 1- $\mu$ m diameter magnetic beads from a fluid flow. The coil on the right is a planar electromagnet actuated with a current of $0.3 \mathrm{~A}$, while the coil on the left is without a current. The width of the coil is $50 \mu \mathrm{m}$. (Reprinted from Choi et al. 2001, with permission from Elsevier)

respect to the field generated by the $\mathrm{Cu}$ winding only. The coil on the right is actuated with a current of $0.3 \mathrm{~A}$ and separates the beads from the flowing solution, while the coil on the left is without a current and attracts no magnetic beads from the flow.

An important difference between the use of a permanent magnet and an electromagnet is the much lower generated magnetic induction of the latter. A permanent magnet easily generates a magnetic induction of $0.5-1 \mathrm{~T}$, while the magnetic induction of a simple planar coil is more in the mTesla range. Following the discussion of Eqs. 2 and 3, one understands that an integrated electromagnet will produce a much smaller magnetic force, so the fluidic flow in the microchannels needs to be strongly limited or the magnetic beads need to pass at a very close distance with the planar coil. Therefore, an interesting idea was to combine a nominally uniform

external magnetic field, to impose a large magnetic moment to the beads, with integrated linear conductors for the generation of a local magnetic field gradient (Tondra et al. 2001). Such a setup allowed for the application of relatively strong and very local applied forces: $0.5 \mathrm{pN}$ for $2.8-\mu \mathrm{m}$ diameter polystyrene particles on the scale of the microfluidic channel width.

In other work, a magnetic separation system was realized using an array of $15-\mu \mathrm{m}$ wide electroplated $\mathrm{Ni}$ posts as filtering elements placed inside microfluidic channels made by soft lithography in poly-(dimethylsiloxane) (PDMS) (Deng et al. 2002). Once magnetized by a magnetic field from an external $\mathrm{NdFeB}$ permanent magnet, these $\mathrm{Ni}$ posts attracted the magnetic field lines and, thereby, generated strong magnetic field gradients that could efficiently trap superparamagnetic beads passing them in a flowing stream of water. These Ni post arrays were also used to separate magnetic beads from non-magnetic beads. Similar work was published on the trapping of magnetic particles flowing through a microfluidic channel realized in PDMS (Forbes et al. 2003); here, the authors have used magnetic "anchors" formed by 4- $\mu \mathrm{m}$ diameter magnetic beads embedded in the sidewall of the microfluidic channel during the PDMS molding process.

\subsection{Transport of magnetic beads}

Magnetic separation is different from magnetic transport in the sense that, in separation, the microbeads are retained (separated) by the action of a magnetic field, but are transported using a liquid flow. In magnetic transport, magnetic forces effectively transport the particles, which is a bigger challenge: it requires magnetic fields and magnetic forces that act on a larger range than necessary for separation, where magnetic beads approach very closely the magnetic actuation region by means of the fluid motion. Manipulation of magnetic beads in general, and transport in particular, is a difficult task, as the magnetic susceptibility $\chi$ of the magnetic beads is rather weak (typically $\chi \leq 1$ ), due to small magnetic core volumes and the demagnetization effects of the particles (see Eq. 2). This explains why, mostly, the large field of (mechanically moving) permanent magnets has been used for the separation, transport, and positioning of the magnetic microbeads (Miltenyi et al. 1990). In an approach towards miniaturization and automation of analytical applications, a system has been proposed in which liquid movement is substituted with magnetically induced movement of magnetic particles (Ostergaard et al. 1999). Fluidic channels were realized on a plastic cartridge of centimeters in size and the magnetic transport was induced by mechanically moving external permanent magnets. In another approach, magnetic particles have been transported over millimeter distances in a microfluidic channel using an array of electromagnets actuated in a four-phase scheme (Joung et al. 2000). Each electromagnet consisted of a $0.3-\mathrm{mm}$ 
diameter magnetic needle core with a wire-wound coil of 300 turns. For coil currents of the order of $0.5 \mathrm{~A}$, forces of $0.1 \mathrm{pN}$ were possible.

Besides these still "macroscopic" transport systems, miniaturized solutions have also been proposed for bead transport, thereby taking full profit from batch microfabrication technologies. Typically, the size of the (micropatterned) magnets determines the spatial range, where appreciable magnetic forces acting on the microbeads exist. Serpentine gold wires micropatterned on silicon substrates have been combined with microfluidic structures realized in PDMS to transport $4.5-\mu \mathrm{m}$ polystyrene-coated magnetic beads (Deng et al. 2001). By engineering the magnetic field generated by different current-carrying wires, a microsystem was realized that could generate local magnetic field maxima that trap the magnetic beads. When the field maxima change locations, the microbeads follow those maxima. The device allowed the precise positioning and transport over 100$\mu \mathrm{m}$ distances in a single actuation event, which is partly due to the presence of a permanent magnet placed in proximity of the microfluidic chip, the role of which, principally, is to enhance the magnetic force by inducing a magnetic moment in the magnetic beads. A microelectromagnet wire matrix, based on two layers of mutually orthogonal arrays of linear wires, has demonstrated magnetic transport of $1-2-\mu \mathrm{m}$ size magnetic particles over $20-\mu \mathrm{m}$ distances in a single actuation event (Lee et al. 2001). This is a typical working range for the magnetic force generated by a current-carrying conductor, when no external permanent magnet is used to induce a magnetic moment to the beads. Figure 10a demonstrates the motion of a group of superparamagnetic particles by the application of currents through different wires of the two-dimensional wire matrix. The wire currents were adjusted so that they continuously move particles by increments that are less than the wire spacing. Figure 10b shows a group of particles that is moving vertically by the matrix over a longer range; Fig. 10c shows how two groups of magnetic particles can be moved diagonally to join in a cluster at a single location. In another approach, a simple planar-coil-array-based magnetic transport system has been proposed in which an individual coil is capable of displacing beads over millimeter distances in a liquid-containing capillary (Rida et al. 2003a). A drastic increase of the magnetic energy and magnetic forces acting on the beads was obtained by placing the complete coil array in a uniform static magnetic field that imposes a permanent magnetic moment to the microbeads. The very small magnetic field (gradient) of a simple planar coil proved to be sufficient to displace $1-\mu \mathrm{m}$ diameter beads over a distance of the order of the coil size. The coils were realized using simple printed circuit board (PCB) technology (100- $\mu \mathrm{m} \mathrm{Cu}$ winding width, 35- $\mu \mathrm{m}$ winding height, 200$\mu \mathrm{m}$ winding pitch) and had a small number of windings $(N=4-10)$. A single coil typically generates a magnetic field gradient of about $5 \mathrm{mT} / \mathrm{mm}$ for a maximum allowed current density of $400 \mathrm{~A} / \mathrm{mm}^{2}$. Arranging adjacent coils with spatial overlap over two layers of the PCB circuit and actuating them in a specific three-phase scheme assured the long-range displacement of the microbeads. Moreover, it was found that these polarized beads formed cylindrical columns with a length of the order of the microfluidic channel size, due to magnetic dipole interactions. This column formation was partly the basis of the strong magnetic force.

An interesting study reported on the magnetic actuation and transport of magnetic beads contained in water droplets that were suspended in a silicone oil solution (Shikida et al. 2004). Such droplet handling can be the basis of a new type of micro-chemical analysis system, where droplets can be magnetically transported thanks to their magnetic bead content, can be fused to trigger chemical reactions, or can be split up in separate droplets. All of these basic actions have been demonstrated by magnetic actuation with a mechanically moving permanent magnet and using 30-nl droplets containing $30-\mu \mathrm{m}$ diameter magnetic beads.

4.5 Magnetic supraparticle structures in microfluidic devices

\subsubsection{Magnetic supraparticle structures}

Most of the magnetic-bead-related phenomena discussed so far were based on the interaction of individual beads with the magnetic field. However, when placed in a field, superparamagnetic particles will acquire a magnetic moment and they will start interacting by means of the magnetic dipole interaction. This interaction induces a spontaneous clustering of the particles into larger, often complicated, structures, which we call magnetic supraparticle structures (SPS). The shape of an SPS depends on the parameters, like the size of the magnetic moment of the microbeads and the magnetic dipolar interaction between different beads. These properties are dependent on the amplitude and frequency of the applied magnetic field, the shape and magnetic content of the beads, the concentration of the magnetic particles in the fluid, the temperature, etc. Despite the complexity of the aggregation process of a magnetic fluid into a SPS, the physical effects of a magnetic field on such a structure are now very well understood (Liu et al. 1995; Flores et al. 1999). When exposed to a strong, continuous magnetic field, the magnetic fluid will rapidly form a cross-linked network. The continuous-field structure is determined by the kinetics of aggregation and particles are prohibited from rearranging to minimize energy as long as the field persists. In contrast, it was found that the application of a pulsed field (square wave alternating between field-on and field-off states) to a magnetic fluid did produce an energetically determined suspension structure (Promislow and Gast 1996; Promislow and Gast 1997). By allowing particle diffusion during the field-off state, a pulsed field enables the minimization of energy through structural rearrangements and the SPS 

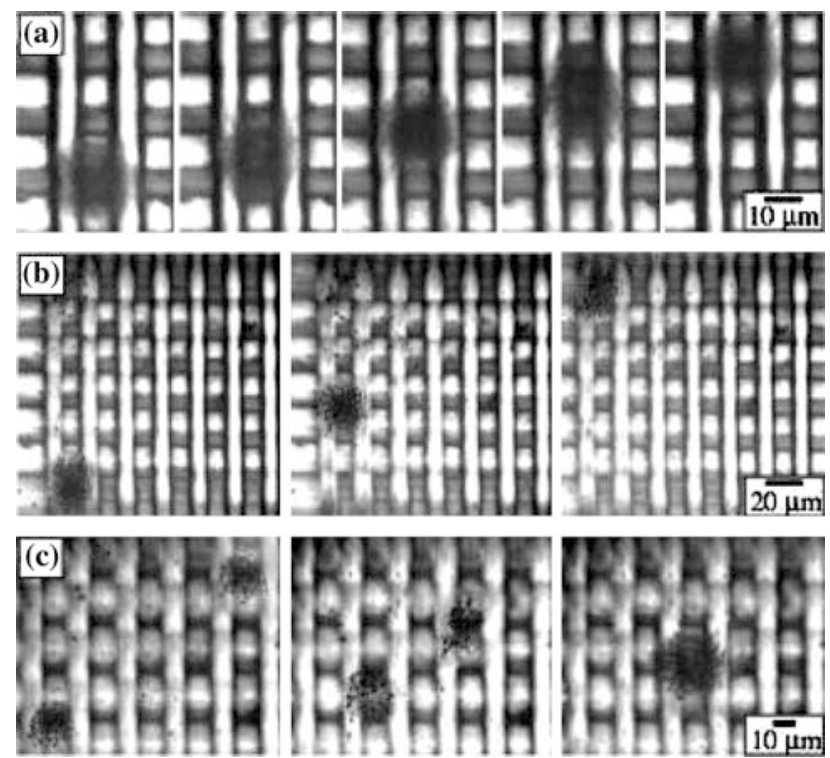

Fig. 10 a Demonstration of moving a group of superparamagnetic particles by application of currents through different wires of a two-dimensional micro-electromagnet wire matrix. The wire currents were adjusted so that they continuously move particles by increments that are less than the wire spacing. b A group of particles is moved vertically by the matrix over a longer range of five wire spacings. $\mathbf{c}$ Two groups of particles are moved diagonally to join in a cluster at a single location (reprinted from Lee et al. 2001, with permission from the American Institute of Physics)

consists of one-dimensional periodic patterns composed of high-concentration regions of magnetic particles ("columns"), aligned in the field direction and sharply separated from low-concentration regions (Wirtz and Fermigier 1994; Liu et al. 1995; Wirtz and Fermigier 1995). Qualitatively, the dependence of the structure complexity on the tuning parameters can be understood from the time that it takes for the particles to aggregate together, which depends on the ratio of the magnetic interaction energy between particles relative to thermal energy. Applying a bi-axial rotating magnetic field, produced by two orthogonal pairs of Helmholtz coils in quadrature, induced a rotation of dipolar chains of superparamagnetic particles and subjected the aggregates to magnetic forces, causing them to rotate within the suspending fluid. It has been shown that, with both scattering dichroism (Melle et al. 2000) and videomicroscopy experiments (Melle et al. 2002), magnetic fluids have the capacity of reducing the size of the structures they are composed of, to decrease their viscous drag while rotating synchronously with the field.

Magnetic assembly of microbead chains onto a surface has been identified as a viable method for printing biological molecules or other nanodevices in aqueous conditions, without using extreme temperatures or harmful chemical solvents (Yellen et al. 2002, 2003). It was shown that superparamagnetic particles assemble on a micropattern of thin ferromagnetic islands, acting as ferromagnetic traps; magnetic bead trapping could be controlled by varying the external magnetic field bias. In addition, a programmable self-assembly method for the placement of two or more different types of superparamagnetic colloidal beads onto lithographically defined magnetic microwell templates has been demonstrated (Yellen and Friedman 2004).

\subsubsection{Separation using magnetic supraparticle structures}

In a previous section, we have discussed the separation of magnetic beads or magnetic-bead-labeled molecules from a liquid flow, using magnetic fields locally applied within the channel. The present section is on the use of magnetic SPS within a microfluidic channel as a porous separation medium for the separation of long DNA molecules. The structure and porosity of the SPS is a strong function of the microfluidic channel dimension, particle properties, applied magnetic field, etc.

Gel electrophoresis is the standard method for the separation of DNA by length. However, the efficiency of gel electrophoresis deteriorates seriously for DNA molecules longer than about 40,000 bp (40 kbp). This phenomenon was understood in terms of electric-fieldinduced aggregation of the DNA by the electric dipoledipole interaction (Mitnik et al. 1995). Slab gel pulsedfield gel electrophoresis or pulsed-field capillary gel electrophoresis, using time-varying drive voltages, can be used to separate the longer chains of DNA (Kim and Morris 1994).

As an alternative, an entropic trap array system with lithographically defined constrictions comparable to the molecular dimension was demonstrated to rapidly separate large DNA fragments using static (DC) electric fields (Han and Craighead 2000). Thick and thin regions ${ }^{4}$ alternated along the channel and DNA molecules were entropically trapped in the thick region and escaped with a characteristic lifetime; longer DNA was found to escape entropic traps faster than shorter ones (Han et al. 1999).

Also, self-assembled magnetic SPS have been used for long DNA separation in microfluidic channels. This represents a convenient solution, since no microlithography is required to define geometrical constrictions that are simply defined by the porous magnetic matrix (Doyle et al. 2002). Figure 11a is a schematic diagram of the microchannel and magnetic coil system. A microchannel with a height of $11 \mu \mathrm{m}$ and defined using PDMS molding was placed in the center of a magnetic coil. The channel is characterized by a pinched injection design with the reservoirs placed at the inner part of the wafer. The chosen configuration guarantees a constant magnetic field (of the order of $10 \mathrm{mT}$ ) along the separation channel. The chip was mounted on an inverted microscope and filled before each run with a solution of $1-\mu \mathrm{m}$

\footnotetext{
${ }^{4} \mathrm{~A}$ thick region is characterized by a channel depth that is of the order of the radius of gyration of the DNA molecule. A thin region has a much smaller depth, which effectively blocks the DNA in the thick region unless it is deformed at the cost of an entropy change.
} 
diameter magnetic microbeads. Figure $11 \mathrm{~b}$ schematically shows the columnar SPS formed by a suspension of superparamagnetic particles in the magnetic field. Intercolumn spacing and porosity of the SPS is a function of the applied magnetic field. Figure 11c shows the fluorescence intensity at $10 \mathrm{~mm}$ from the injection zone versus time, corresponding to a separation of a mixture of $\lambda$-phage DNA and $\lambda$-DNA digested with Xho I (Pharmacia) (the numbers refer to the size of the DNA fragments in kilo-base pairs), at field strengths of $4.8 \mathrm{~V} /$ $\mathrm{cm}$ (right), $7 \mathrm{~V} / \mathrm{cm}$ (middle), and 10 (left) V/cm. Figure $11 \mathrm{~d}$ is a separation of $\lambda$-phage DNA concatemers at a field of $3.2 \mathrm{~V} / \mathrm{cm}$ at conditions identical to those in Figure 11c. Experimental separations using the SPS matrix have been combined with theoretical modeling (Dorfman and Viovy 2004) and, recently, computer-piloted flow control and injection of the experiment resulted in a quantitative and reproducible separation of long DNA by an SPS matrix (Minc et al. 2004).

Fig. 11 a Schematic diagram of the separation microchannel and magnetic coil. The poly(dimethylsiloxane) microchannel was placed in the center of a magnetic coil mounted on an inverted microscope and filled before each run with a solution of $1-\mu \mathrm{m}$ magnetic microbeads. b Columnar structure formed by a suspension of superparamagnetic particles. c Fluorescence intensity at $10 \mathrm{~mm}$ from the injection zone versus time. Separation of a mixture of $\lambda$ phage DNA and $\lambda$-DNA digested with Xho I (Pharmacia) (the numbers refer to the size of the DNA fragments in kilo-base pairs), at field strengths of $4.8 \mathrm{~V} / \mathrm{cm}$ (right), $7 \mathrm{~V} / \mathrm{cm}$ (middle), and 10 (left) $\mathrm{V} / \mathrm{cm}$. d Separation of $\lambda$-phage DNA concatemers at a field of $3.2 \mathrm{~V} / \mathrm{cm}$. Other conditions are identical to those in c. (Reprinted from Doyle et al. 2002, with permission from the American Association for the Advancement of Science)

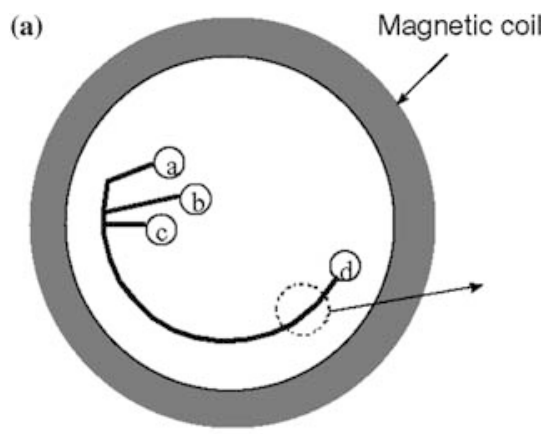

(c)

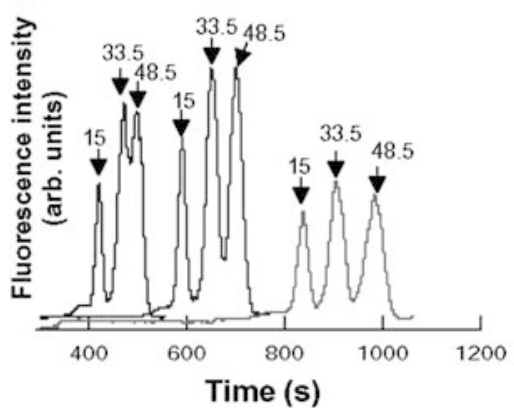

4.5.3 Dynamic manipulation of magnetic supraparticle structures

Besides the use of magnetic SPS as a static stationary phase for separation in microfluidic channels, researchers have also been investigating the potential of manipulating dynamically magnetic particle aggregates for microfluidic applications. Despite its potential for magnetic-bead-based applications, only a few works are reported on the active manipulation of SPS composed from superparamagnetic particles inside microfluidic capillaries. Under the influence of a varying magnetic field generated by a mechanically moving permanent magnet, magnetic SPS composed of $1-2-\mu$ m diameter superparamagnetic beads could be rotated through all axes in a microfluidic channel, without loss of structural form, allowing dynamic micron-scale movement without direct mechanical, electrical, or photonic interactions (Hayes et al. 2001a). A number of potential applications of this phenomenon were mentioned in this paper, like binding biomolecules on the magnetic particles for immuno-assays, studying subcellular biomechanics, and microfluidic mixing in picoliter and femtoliter volumes.

Active fluid mixing was demonstrated in microchannels made in a micromachined microfluidic chip of polymethylmetacrylate (PMMA): mixing was based on the manipulation by a local alternating magnetic field of self-assembled porous structures of magnetic microbeads that are placed over the section of the channel (Rida et al. 2003b; Rida and Gijs 2004a; Rida and Gijs 2004b). Using a sinusoidally varying magnetic field $(1 \mathrm{~Hz}<f<100 \mathrm{~Hz})$, a rotational motion of the SPS was induced, thereby, strongly enhancing the fluid perfusion through the SPS that behaved as a dynamic random porous medium. The localized time-dependent magnetic

(b)
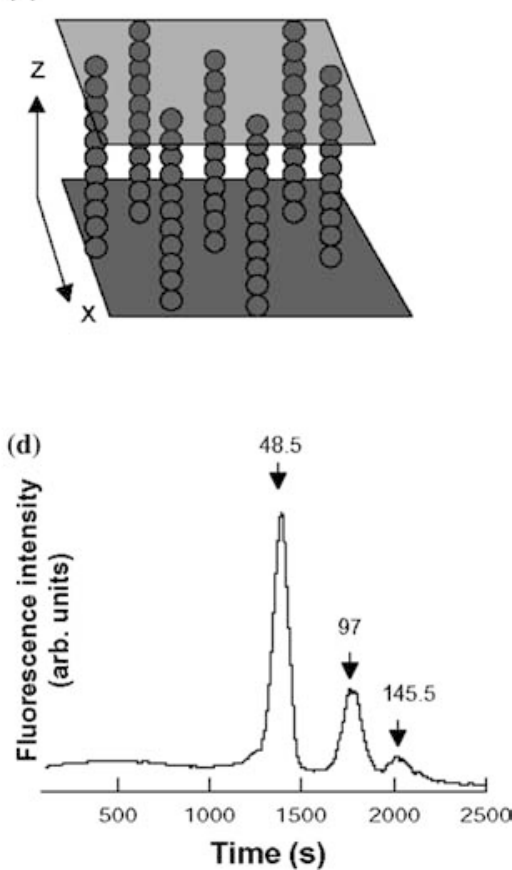
field was generated using a current-fed coil and a soft magnetic yoke structure, which is partly integrated within a plastic microfluidic chip. Figure 12a is a schematic view of the magnetic SPS in a microfluidic channel, obtained using the alternating magnetic field between the magnetic pole tips: the SPS is organized in sheet structures, composed of columnar-like objects and smaller rotating magnetic objects. Figure $12 \mathrm{~b}$ and $\mathrm{c}$ are video images of a dense SPS, in the absence of a liquid flow $\left(v_{0}=0\right)$, at a field oscillation frequency of $f=5 \mathrm{~Hz}$, and field amplitudes of $B_{0}=100 \mathrm{mT}$ and $B_{0}=5 \mathrm{mT}$, respectively. Figure $12 \mathrm{~d}$ and e show the longitudinal (along the $x$ axis) oscillation of the SPS; the images were taken at the most downstream position in the channel at $f=5 \mathrm{~Hz}$, with flow velocity $v_{0}=0.4 \mathrm{~cm} / \mathrm{s}$ and field amplitudes of $B_{0}=25 \mathrm{mT}$ and $B_{0}=20 \mathrm{mT}$, respectively. The mixing is the result of the chaotic splitting of fluid streams through the dynamic and randomly porous structure of the SPS and the relative motion of magnetic entities with respect to the fluid flow. It was quantified by monitoring the fluorescent intensity of initially parallel fluorescent and non-fluorescent laminar streams. A $95 \%$ mixing efficiency over a channel length as small as the channel width $(200 \mu \mathrm{m})$ and at flow rates of $0.5 \mathrm{~cm} / \mathrm{s}$ was obtained, demonstrating the large lateral mass transfer induced by the SPS as a consequence of the highly heterogeneous and dynamic nature of the SPS (Rida and Gijs 2004b).

Besides mixing, the agglomeration of magnetic microbeads into an SPS has also shown to play a role in magnetic transport. The large magnetic bead transport velocities $(10 \mathrm{~mm} / \mathrm{s})$ obtained by actuation using a planar

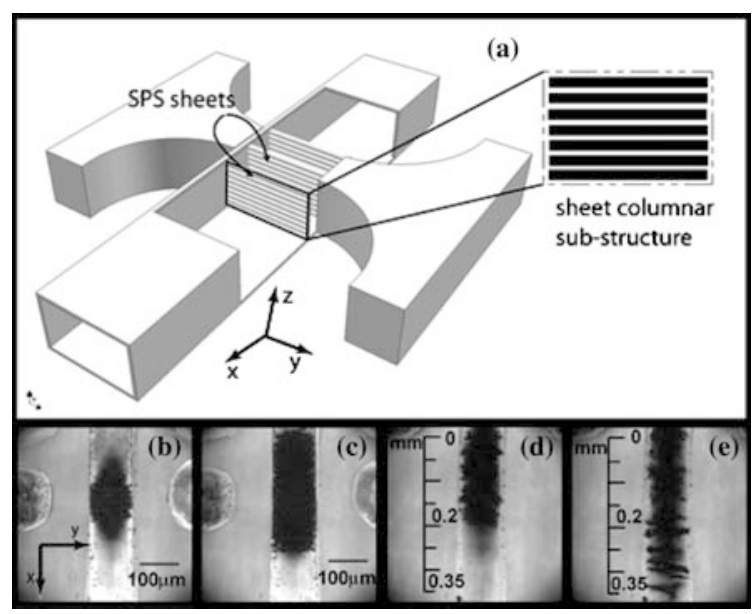

Fig. 12 a Schematic view of a magnetic supraparticle structure (SPS) in a microfluidic channel, obtained by application of an alternating magnetic field between two soft magnetic pole tips sandwiching the channel: the SPS is organized in sheet structures, composed of columnar-like objects. b, c Video images of a dense SPS in the absence of a liquid flow (velocity $v_{0}=0$ ), at a field oscillation frequency of $f=5 \mathrm{~Hz}$ and field amplitudes of $B_{0}=100 \mathrm{mT}$ and $B_{0}=5 \mathrm{mT}$, respectively. d, e The longitudinal (along the x-axis) oscillation of the SPS; images are taken at the most downstream position in the channel at $f=5 \mathrm{~Hz}$, with flow velocity $v_{0}=0.4 \mathrm{~cm} / \mathrm{s}$ and field amplitudes of $B_{0}=25 \mathrm{mT}$ and $B_{0}=$ $20 \mathrm{mT}$, respectively coil array placed in a uniform magnetostatic field could only be explained by the formation of columnar magnetic objects with a strongly enhanced magnetic moment and corresponding magnetic energy (Rida et al. 2003a).

\section{Conclusions and future prospects}

We have given an update on recent advances in the handling, manipulation, and detection of magnetic beads in microfluidic systems. Starting from the physics of magnetic actuation and the peculiar properties of magnetic nanoparticles and microparticles, their use in "classical" (i.e., non-microfluidic) biomedical applications, like magnetic separation, immuno-assays, MRI, drug delivery, and hyperthermia, was first discussed. Probably the most prominent advantage of magnetic beads over other solid phases lies in the fact that the particles can be magnetically probed and manipulated using permanent magnets or electromagnets, independent of normal chemical or biological processes. Some of the classical applications, like magnetic separation, have already found their way down to miniaturized fluidic or "lab-on-a-chip" systems that strongly limit the consumption of samples and reagents; in such systems, magnetic beads effectively provided a chemically active substrate with a large surface-to-volume ratio.

We hope to have shown that this novel area of miniaturized applications of magnetic beads offers many exciting possibilities for future developments. It is a highly multidisciplinary area, requiring a range of scientific knowledge, from inorganic chemistry involved in the preparation of the magnetic beads, through biochemistry and medical science to allow for their functionalization and, of course, the basic physics of magnetism and magnetic materials. What is remarkable is that nearly all important functions in a bio-assay can be realized using magnetic beads: sample purification, providing a solid substrate to the sample, mixing, labeling, manipulation and transport, and, finally, separation. Therefore, magnetic assays, especially those employing magnetic nanoparticles as specific labels, will certainly find more applications in the near future. Magnetic labeling is more universal and robust than fluorescent labeling, in the sense that the magnetism of the particles cannot be quenched at normal working temperatures. The progress in this area will be supported by the further development of magnetic field sensor hardware technology, which will allow the detection of one or just a few magnetic labels. Extremely promising is the fact that increasingly smaller single beads can be detected by correctly scaling down all the dimensions of the detection system. Ultra-small nanometric beads offer the potential to be of the same size or smaller than the biomolecules attached to them, thereby, providing minimum disturbance to chemical and biological processes and benefitting from decreased diffusion times. Combining such particles and their detection system with the recently developed magnetic manipulation 
techniques could result in highly integrated bio-assay systems in which all functions (from sample treatment to read-out) could be extremely miniaturized.

It is now generally recognized that nanotechnologies and biosciences will be one of the leading and most promising areas of research and development in the $21 \mathrm{st}$ century. I hope this review made clear that magnetic beads could play a very important role in these developments.

Acknowledgements The author gratefully acknowledges Amar Rida, Victor Fernandez, Hicham Majd, Smail Hadjidj, Ulrike Lehmann, Virendra Parashar, and Caroline Vandevyver for useful discussions and their collaboration in the EPFL magnetic bead handling project.

\section{References}

Ahn CH, Allen MG, Trimmer W, Jun YN, Erramilli S (1996) A fully integrated micromachined magnetic particle separator. J Microelectromech Syst 5(3):151-158

Alexiou C (2001) Target tumor therapy with "magnetic drug targeting": therapeutic efficacy and biokinetic study of ferrofluid bound mitoxantrone. Clin Cancer Res 7(11):257

Auroux PA, Iossifidis D, Reyes DR, Manz A (2002) Micro total analysis systems. II: analytical standard operations and applications. Anal Chem 74(12):2637-2652

Baibich MN, Broto JM, Fert A, Vandau FN, Petroff F, Eitenne P, Creuzet G, Friederich A, Chazelas J (1988) Giant magnetoresistance of $(001) \mathrm{Fe} /(001) \mathrm{Cr}$ magnetic superlattices. Phys Rev Lett 61(21):2472-2475

Baselt DR, Lee GU, Natesan M, Metzger SW, Sheehan PE, Colton RJ (1998) A biosensor based on magnetoresistance technology. Biosens Bioelectron 13(7-8):731-739

Bauer LA, Birenbaum NS, Meyer GJ (2004) Biological applications of high aspect ratio nanoparticles. J Mater Chem 14(4):517-526

Bergemann C, Muller-Schulte D, Oster J, Brassard L, Lubbe AS (1999) Magnetic ion-exchange nano- and microparticles for medical, biochemical and molecular biological applications. J Magnetism Magn Mater 194(1-3):45-52

Besse PA, Boero G, Demierre M, Pott V, Popovic R (2002) Detection of a single magnetic microbead using a miniaturized silicon Hall sensor. Appl Phys Lett 80(22):4199-4201

Blankenstein G, Larsen UD (1998) Modular concept of a laboratory on a chip for chemical and biochemical analysis. Biosens Bioelectron 13(3-4):427-438

Cavalier R, Ciocatto EC, Giovanel Bc, Heidelbe C, Johnson RO, Margotti M, Mondovi B, Moricca G, Rossifan A (1967) Selective heat sensitivity of cancer cells - biochemical and clinical studies. Cancer 20(9):1351

Chiem N, Harrison DJ (1997) Microchip-based capillary electrophoresis for immunoassays: analysis of monoclonal antibodies and theophylline. Anal Chem 69(3):373-378

Chikazumi S (1964) Physics of magnetism. R.E. Krieger Publishing Co., Malabar, Florida

Choi JW, Ahn CH, Bhansali S, Henderson HT (2000). A new magnetic bead-based, filterless bio-separator with planar electromagnet surfaces for integrated bio-detection systems. Sens Actuat B Chem 68(1-3):34-39

Choi JW, Liakopoulos TM, Ahn CH (2001) An on-chip magnetic bead separator using spiral electromagnets with semi-encapsulated permalloy. Biosens Bioelectron 16(6):409-416

Choi JW, Oh KW, Thomas JH, Heineman WR, Halsall HB, Nevin JH, Helmicki AJ, Henderson HT, Ahn CH (2002) An integrated microfluidic biochemical detection system for protein analysis with magnetic bead-based sampling capabilities. Lab Chip 2(1):27-30
Coehoorn R, Prins MWJ (2003) Patent application WO 03/054523 A2

Deng T, Whitesides GM, Radhakrishnan M, Zabow G, Prentiss M (2001) Manipulation of magnetic microbeads in suspension using micromagnetic systems fabricated with soft lithography. Appl Phy Lett 78(12):1775-1777

Deng T, Prentiss M, Whitesides GM (2002) Fabrication of magnetic microfiltration systems using soft lithography. Appl Phys Lett 80(3):461-463

Dieny B, Speriosu VS, Metin S, Parkin SSP, Gurney BA, Baumgart P, Wilhoit DR (1991) Magnetotransport properties of magnetically soft spin-valve structures. J Appl Phys 69(8):47744779

Dorfman KD, Viovy JL (2004) Semiphenomenological model for the dispersion of DNA during electrophoresis in a microfluidic array of posts. Phys Rev E 69(1):011901

Doyle PS, Bibette J, Bancaud A, Viovy JL (2002) Self-assembled magnetic matrices for DNA separation chips. Science 295(5563):2237

Dunnill P, Lilly MD (1974) Purification of enzymes using magnetic bio-affinity materials. Biotechnol Bioeng 16(7):987-990

Dynal Biotech product catalogue (2004) Dynal Biotech, Oslo, Norway, home page at http://www.dynal.no

Ebner AD, Ritter JA (2001) New correlation for the capture cross section in high-gradient magnetic separation. AiChE J 47(2):303-313

Edelstein RL, Tamanaha CR, Sheehan PE, Miller MM, Baselt DR, Whitman LJ, Colton RJ (2000) The BARC biosensor applied to the detection of biological warfare agents. Biosens Bioelectron 14(10-11):805-813

Ejsing L, Hansen MF, Menon AK, Ferreira HA, Graham DL, Freitas PP (2004) Planar Hall effect sensor for magnetic microand nanobead detection. Appl Phys Lett 84(23):4729-4731

Fan ZH, Mangru S, Granzow R, Heaney P, Ho W, Dong QP, Kumar R (1999) Dynamic DNA hybridization on a chip using paramagnetic beads. Anal Chem 71(21):4851-4859

Flores GA, Liu J, Mohebi M, Jamasbi N (1999) Field-induced columnar and bent-wall-like patterns in a ferrofluid emulsion. Int J Mod Phys B 13(14-16):2093-2100

Forbes ZG, Yellen BB, Barbee KA, Friedman G (2003) An approach to targeted drug delivery based on uniform magnetic fields. IEEE Trans Magn 39(5):3372-3377

Freitas PP, Silva F, Oliveira NJ, Melo LV, Costa L, Almeida N (2000) Spin valve sensors. Sens Actuat A Phys 81(1-3):2-8

Gordon RT, Hines JR, Gordon D (1979) Intracellular hyperthermia: a biophysical approach to cancer treatment via intracellular temperature and biophysical alterations. Med Hypotheses 5(1):83-102

Graham DL, Ferreira HA, Freitas PP, Cabral JMS (2003) High sensitivity detection of molecular recognition using magnetically labelled biomolecules and magnetoresistive sensors. Biosens Bioelectron 18(4):483-488

Grieve K, Mulvaney P, Grieser F (2000) Synthesis and electronic properties of semiconductor nanoparticles/quantum dots. Curr Opin Colloid Interface Science 5(1-2):168-172

Gruttner C, Teller J (1999) New types of silica-fortified magnetic nanoparticles as tools for molecular biology applications. J Magnetism Magn Mater 194(1-3):8-15

Gruttner C, Rudershausen S, Teller J (2001) Improved properties of magnetic particles by combination of different polymer materials as particle matrix. J Magnetism Magn Mater 225(12): $1-7$

Hadd AG, Raymond DE, Halliwell JW, Jacobson SC, Ramsey JM (1997) Microchip device for performing enzyme assays. Anal Chem 69(17):3407-3412

Hafeli UO, Pauer GJ (1999) In vitro and in vivo toxicity of magnetic microspheres. J Magnetism Magn Mater 194(1-3):7682

Hafeli UO, Sweeney SM, Beresford BA, Sim EH, Macklis RM (1994) Magnetically directed poly(lactic acid) Y-90 microspheres - novel agents for targeted intracavitary radiotherapy. J Biomed Mater Res 28(8):901-908 
Haik Y, Pai V, Chen CJ (1999) Development of magnetic device for cell separation. J Magnetism Magn Mater 194(1-3):254-261

Han J, Craighead HG (2000) Separation of long DNA molecules in a microfabricated entropic trap array. Science 288(5468):10261029

Han J, Turner SW, Craighead HG (1999) Entropic trapping and escape of long DNA molecules at submicron size constriction. Phys Rev Lett 83(8):1688-1691

Hancock JP, Kemshead JT (1993) A rapid and highly selective approach to cell separations using an immunomagnetic colloid. J Immunol Meth 164(1):51-60

Hartmann U (1999) Magnetic multilayers and giant magnetoresistance. Springer, Berlin Heidelberg New York

Hatch GP, Stelter RE (2001) Magnetic design considerations for devices and particles used for biological high-gradient magnetic separation (HGMS) systems. J Magnetism Magn Mater 225(12):262-276

Hayes MA, Polson NA, Garcia AA (2001a) Active control of dynamic supraparticle structures in microchannels. Langmuir 17(9):2866-2871

Hayes MA, Polson NA, Phayre AN, Garcia AA (2001b) Flowbased microimmunoassay. Anal Chem 73(24):5896-5902

Hergt R, Andra W, d'Ambly CG, Hilger I, Kaiser WA, Richter U, Schmidt HG (1998) Physical limits of hyperthermia using magnetite fine particles. IEEE Trans Magn 34(5):3745-3754

Hoffmann C, Franzreb M (2004) A novel repulsive-mode highgradient magnetic separator. II: separation model. IEEE Trans Magn 40(2):462-468

Ikeda N, Hayashida O, Kameda H, Ito H, Matsuda T (1994) Experimental study on thermal damage to dog normal brain. Int J Hyperthermia 10(4):553-561

Jiang GF, Harrison DJ (2000) mRNA isolation in a microfluidic device for eventual integration of cDNA library construction. Analyst 125(12):2176-2179

Jordan A, Scholz R, Maier-Hauff K, Johannsen M, Wust P, Nadobny J, Schirra H, Schmidt H, Deger S, Loening S, Lanksch W, Felix R (2001) Presentation of a new magnetic field therapy system for the treatment of human solid tumors with magnetic fluid hyperthermia. J Magnetism Magn Mater 225(1-2):118126

Joung J, Shen J, Grodzinski P (2000) Micropumps based on alternating high-gradient magnetic fields. IEEE Trans Magn 36(4):2012-2014

Kawaguchi H (2000) Functional polymer microspheres. Prog Polym Sci 25(8):1171-1210

Kelley JR (1977) US patent 4019994

Kim YS, Morris MD (1994) Separation of nucleic acids by capillary electrophoresis in cellulose solutions with mono- and bisintercalating dyes. Anal Chem 66(7):1168-1174

Kim DK, Zhang Y, Kehr J, Klason T, Bjelke B, Muhammed M (2001a) Characterization and MRI study of surfactant-coated superparamagnetic nanoparticles administered into the rat brain. J Magnetism Magn Mater 225(1-2):256-261

Kim DK, Zhang Y, Voit W, Kao KV, Kehr J, Bjelke B, Muhammed M (2001b) Superparamagnetic iron oxide nanoparticles for bio-medical applications. Scripta Materialia 44(89):1713-1717

Kim DK, Mikhaylova M, Wang FH, Kehr J, Bjelke B, Zhang Y, Tsakalakos T, Muhammed M (2003) Starch-coated superparamagnetic nanoparticles as MR contrast agents. Chem Mater 15(23):4343-4351

Kleiber J, Walter T, Harttig H, Lesniak C, Mennig M, Riedling M, Schmidt H (2001) US patent 6255477B1

Kondo A, Kamura H, Higashitani K (1994) Development and application of thermosensitive magnetic immunomicrospheres for antibody purification. Appl Microbiol Biotechnol 41(1):99105

Koneracka M, Kopcansky P, Timko M, Ramchand CN (2002) Direct binding procedure of proteins and enzymes to fine magnetic particles. J Magnetism Magn Mater 252(1-3):409411
Kourilov V, Steinitz M (2002) Magnetic-bead enzyme-linked immunosorbent assay verifies adsorption of ligand and epitope accessibility. Anal Biochem 311(2):166-170

Koutny LB, Schmalzing D, Taylor TA, Fuchs M (1996) Microchip electrophoretic immunoassay for serum cortisol. Anal Chem 68(1):18-22

Kruis FE, Fissan H, Peled A (1998) Synthesis of nanoparticles in the gas phase for electronic, optical and magnetic applications - a review. J Aerosol Sci 29(5-6):511-535

Lagae L, Wirix-Speetjens R, Das J, Graham D, Ferreira H, Freitas PPF, Borghs G, De Boeck J (2002) On-chip manipulation and magnetization assessment of magnetic bead ensembles by integrated spin-valve sensors. J Appl Phys 91(10):7445-7447

Landfester K, Ramirez LP (2003) Encapsulated magnetite particles for biomedical application. J Phys Condensed Matter 15(15):S1345-S1361

Lawaczeck R, Bauer H, Frenzel T, Hasegawa M, Ito Y, Kito K, Miwa N, Tsutsui H, Volger H, Weinmann HJ (1997) Magnetic iron oxide particles coated with carboxydextran for parenteral administration and liver contrasting - pre-clinical profile of $\mathrm{SH}$ U555A. Acta Radiologica 38(4):584-597

Lee JW, Isobe T, Senna M (1996) Magnetic properties of ultrafine magnetite particles and their slurries prepared via in-situ precipitation. Colloids Surf A Physicochem Eng Aspects 109:121127

Lee GU, Metzger S, Natesan M, Yanavich C, Dufrene YF (2000) Implementation of force differentiation in the immunoassay. Anal Biochem 287(2):261-271

Lee CS, Lee H, Westervelt RM (2001) Microelectromagnets for the control of magnetic nanoparticles. Appl Phys Lett 79(20):33083310

Leslie-Pelecky DL, Rieke RD (1996) Magnetic properties of nanostructured materials. Chem Mater 8(8):1770-1783

Li GX, Wang SX (2003) Analytical and micromagnetic modeling for detection of a single magnetic microbead or nanobead by spin valve sensors. IEEE Trans Magn 39(5):3313-3315

Li GX, Joshi V, White RL, Wang SX, Kemp JT, Webb C, Davis RW, Sun SH (2003) Detection of single micron-sized magnetic bead and magnetic nanoparticles using spin valve sensors for biological applications. J Appl Phys 93(10):75577559

Lin JC, Wang YJ (1987) Interstitial microwave antennas for thermal therapy. Int J Hyperthermia 3(1):37-47

Liu J, Lawrence M, Wu A, Ivey ML, Flores GA, Javier K, Bibette J, Richard J (1995) Field-induced structures in ferrofluid emulsions. Phys Rev Lett 74(14):2828-2831

Liu RH, Yang JN, Lenigk R, Bonanno J, Grodzinski P (2004) Selfcontained, fully integrated biochip for sample preparation, polymerase chain reaction amplification, and DNA microarray detection. Anal Chem 76(7):1824-1831

Lubbe AS, Alexiou C, Bergemann C (2001). Clinical applications of magnetic drug targeting. J Surg Res 95(2):200-206

Madou MJ (2002). Fundamentals of microfabrication: the science of miniaturization, 2nd edn. CRC Press, Boca Raton, Florida

Manz A, Graber N, Widmer HM (1990) Miniaturized total chemical analysis systems: a novel concept for chemical sensing. Sens Actuat B Chem 1(1-6):244-248

McCloskey KE, Chalmers JJ, Zborowski M (2003a) Magnetic cell separation: characterization of magnetophoretic mobility. Anal Chem 75(24):6868-6874

McCloskey KE, Moore LR, Hoyos M, Rodriguez A, Chalmers JJ, Zborowski M (2003b) Magnetophoretic cell sorting is a function of antibody binding capacity. Biotechnol Progr 19(3):899907

Melle S, Fuller GG, Rubio MA (2000) Structure and dynamics of magnetorheological fluids in rotating magnetic fields. Phys Rev E 61(4):4111-4117

Melle S, Calderon OG, Rubio MA, Fuller GG (2002) Rotational dynamics in dipolar colloidal suspensions: video microscopy experiments and simulations results. J Non-Newton Fluid Mech 102(2):135-148 
Miller MM, Sheehan PE, Edelstein RL, Tamanaha CR, Zhong L, Bounnak S, Whitman LJ, Colton RJ (2001) A DNA array sensor utilizing magnetic microbeads and magnetoelectronic detection. J Magnetism Magn Mater 225(1-2):138-144

Miltenyi S, Muller W, Weichel W, Radbruch A (1990) High gradient magnetic cell separation with MACS. Cytometry 11(2):231-238

Minc N, Futterer C, Dorfman KD, Bancaud A, Gosse C, Goubault C, Viovy JL (2004) Quantitative microfluidic separation of DNA in self-assembled magnetic matrixes. Anal Chem 76(13):3770-3776

Mitnik L, Heller C, Prost J, Viovy JL (1995) Segregation in DNA solutions induced by electric fields. Science 267(5195):219-222

Molday RS (1984) US patent 4452773

Moller W, Nemoto I, Heyder J (2003) Effect of magnetic bead agglomeration on cytomagnetometric measurements. IEEE Trans Nanobiosci 2(4):247-254

Moroz P, Jones SK, Gray BN (2002) Magnetically mediated hyperthermia: current status and future directions. Int $\mathbf{J}$ Hyperthermia 18(4):267-284

Mosbach K, Andersson L (1977) Magnetic ferrofluids for preparation of magnetic polymers and their application in affinity chromatography. Nature 270(5634):259-261

Murray CB, Kagan CR and Bawendi MG (2000) Synthesis and characterization of monodisperse nanocrystals and closepacked nanocrystal assemblies. Annu Rev Mater Sci 30:545610

Nedelcu S, Watson JHP (2002) Magnetic separator with transversally magnetised disk permanent magnets. Miner Eng 15(5):355-359

Ogiue-Ikeda M, Sato Y, Ueno S (2003) A new method to destruct targeted cells using magnetizable beads and pulsed magnetic force. IEEE Transact Nanobiosci 2(4):262-265

Ostergaard S, Blankenstein G, Dirac H, Leistiko O (1999) A novel approach to the automation of clinical chemistry by controlled manipulation of magnetic particles. J Magnetism Magn Mater 194(1-3):156-162

Pankhurst QA, Connolly J, Jones SK, Dobson J (2003) Applications of magnetic nanoparticles in biomedicine. J Phys D-Appl Phys 36(13):R167-R181

Papell SS (1965) US patent 3215572

Promislow JHE, Gast AP (1996) Magnetorheological fluid structure in a pulsed magnetic field. Langmuir 12(17):4095-4102

Promislow JHE, Gast AP (1997) Low-energy suspension structure of a magnetorheological fluid. Phys Rev E 56(1):642-651

Rashkovetsky LG, Lyubarskaya YV, Foret F, Hughes DE, Karger BL (1997) Automated microanalysis using magnetic beads with commercial capillary electrophoretic instrumentation. J Chromatogr A 781(1-2):197-204

Reimers GW, Khalfalla SE (1974) US patent 3843540

Rembaum A (1981) US patent 4267234

Reyes DR, Iossifidis D, Auroux PA, Manz A (2002) Micro total analysis systems. I: introduction, theory, and technology. Anal Chem 74(12):2623-2636

Rheinlander T, Kotitz R, Weitschies W, Semmler W (2000) Magnetic fractionation of magnetic fluids. J Magnetism Magn Mater 219(2):219-228

Rida A, Gijs MAM (2004a) Dynamics of magnetically retained supraparticle structures in a liquid flow. Appl Phys Lett, to be published

Rida A, Gijs MAM (2004b) Manipulation of self-assembled structures of magnetic beads for microfluidic mixing and assaying. Anal Chem DOI: 10.1021/ac049415j

Rida A, Fernandez V, Gijs MAM (2003a) Long-range transport of magnetic microbeads using simple planar coils placed in a uniform magnetostatic field. Appl Phys Lett 83(12):23962398

Rida A, Lehnert T, Gijs MAM (2003b) Microfluidic mixer using magnetic beads. In: Proceedings of the 7 th international conference on miniaturized chemical and biochemical analysis systems ( $\mu$ TAS 2003), Squaw Valley, California, 5-9 October 2003, pp 579-582
Rife JC, Miller MM, Sheehan PE, Tamanaha CR, Tondra M, Whitman LJ (2003) Design and performance of GMR sensors for the detection of magnetic microbeads in biosensors. Sensor Actuat A-Phys 107(3):209-218

Roger J, Pons JN, Massart R, Halbreich A, Bacri JC (1999) Some biomedical applications of ferrofluids. Eur Phys J Appl Phys 5(3):321-325

Romano G, Sacconi L, Capitanio M, Pavone FS (2003) Force and torque measurements using magnetic micro beads for single molecule biophysics. Optics Commun 215(4-6):323-331

Rosensweig RE (2002) Heating magnetic fluid with alternating magnetic field. J Magnetism Magn Mater 252(1-3):370-374

Ruehm SG, Corot C, Vogt P, Kolb S, Debatin JF (2001) Magnetic resonance imaging of atherosclerotic plaque with ultrasmall superparamagnetic particles of iron oxide in hyperlipidemic rabbits. Circulation 103(3):415-422

Safarik I, Safarikova M (2002) Magnetic nanoparticles and biosciences. Monatsh Chem 133(6):737-759

Sandre O, Browaeys J, Perzynski R, Bacri JC, Cabuil V, Rosensweig RE (1999) Assembly of microscopic highly magnetic droplets: magnetic alignment versus viscous drag. Phys Rev E 59(2):1736-1746

Sato K, Tokeshi M, Odake T, Kimura H, Ooi T, Nakao M, Kitamori T (2000) Integration of an immunosorbent assay system: analysis of secretory human immunoglobulin A on polystyrene beads in a microchip. Anal Chem 72(6):1144-1147

Semelka RC, Helmberger TKG (2001) Contrast agents for MR imaging of the liver. Radiology 218(1):27-38

Shikida M, Inouchi K, Honda H, Sato K (2004) Magnetic handling of droplet in micro chemical analysis system utilizing surface tension and wettability. In: Proceedings of the 17th IEEE international conference on micro electro mechanical systems (MEMS 2004), Maastricht, The Netherlands, January 2004, pp 359-362

Shinkai M (2002) Functional magnetic particles for medical application. J Biosci Bioeng 94(6):606-613

Singer JM (1987) In: El-Asser MS, Fitch RM (eds) Future directions in polymer colloids. NATO ASI series, series E: applied sciences, no. 138. Published in cooperation with NATO Scientific Affairs Division by Nijhoff, Dordrecht, The Netherlands

Stauffer PR, Cetas TC, Fletcher AM, Deyoung DW, Dewhirst MW, Oleson JR, Roemer RB (1984) Observations on the use of ferromagnetic implants for inducing hyperthermia. IEEE Trans Biomed Eng 31(1):76-90

Suzuki M, Shinkai M, Kamihira M, Kobayashi T (1995) Preparation and characteristics of magnetite-labeled antibody with the use of Poly(Ethylene Glycol) derivatives. Biotechnol Appl Biochem 21:335-345

Swihart MT (2003) Vapor phase synthesis of nanoparticles. Curr Opin Colloid Interface Sci 8(1):127-133

Tiwari A, Punshon G, Kidane A, Hamilton G, Seifalian AM (2003) Magnetic beads (Dynabead ${ }^{\mathrm{TM}}$ ) toxicity to endothelial cells at high bead concentration: implication for tissue engineering of vascular prosthesis. Cell Biol Toxicol 19(5):265-272

Todd P, Cooper RP, Doyle JF, Dunn S, Vellinger J, Deuser MS (2001) Multistage magnetic particle separator. J Magnetism Magn Mater 225(1-2):294-300

Tondra M, Granger M, Fuerst R, Porter M, Nordman C, Taylor J, Akou S (2001) Design of integrated microfluidic device for sorting magnetic beads in biological assays. IEEE Trans Magn 37(4):2621-2623

Tondra M, Porter M, Lipert RJ (2000) A model for detection of immobilized superparamagnetic nanosphere assay labels using giant magnetoresistive sensors. J Vac Sci Technol A-Vac Surfaces Films 18(4):1125-1129

Trindade T, O'Brien P, Pickett NL (2001) Nanocrystalline semiconductors: synthesis, properties, and perspectives. Chem Mater 13(11):3843-3858

Undisclosed inventors (2001) European patent 1154443A1

Valberg PA, Butler JP (1987) Magnetic particle motions within living cells-physical theory and techniques. Biophys J 52(4):537-550 
Verpoorte E (2003) Beads and chips: new recipes for analysis. Lab Chip 3(4):60N-68 N

Vilkner T, Janasek D, Manz A (2004) Micro total analysis systems: recent developments. Anal Chem 76(12):3373-3385

Wang H, Zhu Y, Boyd C, Luo WL, Cebers A, Rosensweig RE (1994) Periodic branched structures in a phase-separated magnetic colloid. Phys Rev Lett 72(12):1929-1932

Wang N, Butler JP, Ingber DE (1993) Mechanotransduction across the cell surface and through the cytoskeleton. Science 260(5111):1124-1127

White FM (1999) Fluid mechanics, 4th edn. McGraw-Hill, New York

Wilhelm C, Gazeau F, Roger J, Pons JN, Bacri JC (2002) Interaction of anionic superparamagnetic nanoparticles with cells: kinetic analyses of membrane adsorption and subsequent internalization. Langmuir 18(21):8148-8155

Williams PS, Zborowski M, Chalmers JJ (1999) Flow rate optimization for the quadrupole magnetic cell sorter. Anal Chem 71(17):3799-3807
Wirtz D, Fermigier M (1994) One-dimensional patterns and wavelength selection in magnetic fluids. Phys Rev Lett 72(14):2294-2297

Wirtz D, Fermigier M (1995) Periodic structures and substructures in magnetic suspensions. Langmuir 11(2):398-400

Yellen B, Friedman G, Feinerman A (2002) Analysis of interactions for magnetic particles assembling on magnetic templates. J Appl Phys 91(10):8552-8554

Yellen B, Friedman G, Feinerman A (2003) Printing superparamagnetic colloidal particle arrays on patterned magnetic film. J Appl Phys 93(10):7331-7333

Yellen BB, Friedman G (2004) Programmable assembly of colloidal particles using magnetic microwell templates. Langmuir 20(7):2553-2559

Zborowski M, Sun LP, Moore LR, Williams PS, Chalmers JJ (1999) Continuous cell separation using novel magnetic quadrupole flow sorter. J Magnetism Magn Mater 194(1-3):224-230 